\title{
A Construction of Observables for AKSZ Sigma Models
}

\author{
PAVEL MNEV \\ Institut für Mathematik, Universität Zürich, Winterthurerstrasse 190, 8057 Zurich, \\ Switzerland.e-mail: pmnev@pdmi.ras.ru
}

Received: 7 June 2014 / Revised: 10 July 2015 / Accepted: 22 July 2015

Published online: 5 August 2015 - (C) Springer Science+Business Media Dordrecht 2015

\begin{abstract}
A construction of gauge-invariant observables is suggested for a class of topological field theories, the AKSZ sigma models. The observables are associated to extensions of the target $Q$-manifold of the sigma model to a $Q$-bundle over it with additional Hamiltonian structure in fibers.
\end{abstract}

Mathematics Subject Classification. 57R56, 58A50, 81T70.

Keywords. Topological field theory, $Q$-manifolds, observables, Batalin-Vilkovisky formalism.

\section{Contents}

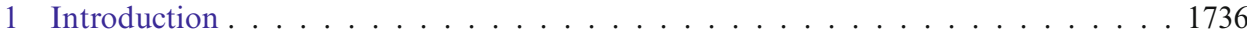

1.1 LOGIC OF THE CONSTRUCTION . . . . . . . . . . . . . . . 1738

1.2 PLAN OF THE PAPER . . . . . . . . . . . . . . . . . . . . . 1739

2 AKSZ Reminder . . . . . . . . . . . . . . . . . . . . 1741

2.1 TARGET DATA . . . . . . . . . . . . . . . . . . 1741

2.2 THE AKSZ SIGMA MODEL . . . . . . . . . . . . . . . . . 1741

2.3 EXAMPLES . . . . . . . . . . . . . . . . . . . . . 1744

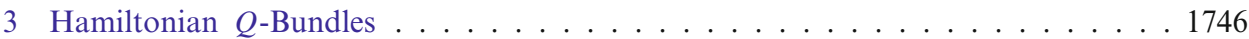

$3.1 Q$-BUNDLES REMINDER . . . . . . . . . . . . . . . . 1746

3.2 TRIVIAL HAMILTONIAN $Q$-BUNDLES $\ldots \ldots \ldots \ldots$. . . . . . . . 1747

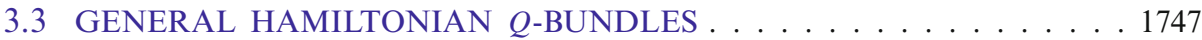

3.4 EXAMPLES . . . . . . . . . . . . . . . . . . . . . 1748

4 BV Pre-observables and Observables . . . . . . . . . . . . . . . . 1750

4.1 PRE-OBSERVABLES AND OBSERVABLES FOR A CLASSICAL AMBI-

ENT BV THEORY . . . . . . . . . . . . . . . . . . . 1751

4.2 PRE-OBSERVABLES AND OBSERVABLES FOR A QUANTUM AMBI-

ENT BV THEORY . . . . . . . . . . . . . . . . . . 1755

5 AKSZ Pre-observable Associated to a Hamiltonian $Q$-Bundle . . . . . . . . 1758

6 From Pre-observables to Observables . . . . . . . . . . . . . . . . . . . 1762

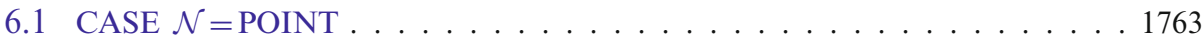

6.2 CASE OF ONE-DIMENSIONAL OBSERVABLES . . . . . . . . . . . . . 1764

6.3 TORSION-LIKE OBSERVABLES . . . . . . . . . . . . . . . . . 1766 
7 Examples of Observables . . . . . . . . . . . . . . . . . . . . . 1771

7.1 OBSERVABLES WITH $\mathcal{N}$ A POINT . . . . . . . . . . 1771

7.2 WILSON LOOP OBSERVABLE IN CHERN-SIMONS THEORY . . . . 1772

7.3 "WILSON LOOPS" IN POISSON SIGMA MODEL . . . . . . . . . . . 1774

7.4 TORSION OBSERVABLES IN CHERN-SIMONS THEORY $\ldots \ldots \ldots . . .1775$

7.5 A CODIMENSION 2 (PRE-)OBSERVABLE IN BF THEORY . . . . . . 1778

8 Final Remarks . . . . . . . . . . . . . . . . . . . . . . . . . 1780

8.1 EXTENSION TO SOURCE MANIFOLDS WITH BOUNDARY . . . 1780

References . . . . . . . . . . . . . . . . . . . . . . . . . 1782

\section{Introduction}

The interest in observables in topological field theories is largely due to the applications in algebraic topology, where expectation values of observables are known to yield (at least in some examples) invariants of knots and links under ambient isotopy or, more generally, cohomology classes of spaces of embeddings. The seminal example here was given in the work of Witten [20], where the expectation value of the Wilson loop observable in Chern-Simons theory with gauge group $S U(2)$, associated to a knot in the 3 -sphere, was found out to yield the Jones polynomial of the knot.

Given a diffeomorphism-invariant action $S_{\Sigma}$ of a topological field theory on a manifold $\Sigma$ with space of fields $F_{\Sigma}$, one is particularly interested in observables $O \in C^{\infty}\left(F_{\Sigma}\right)$ associated to embedded submanifolds $\mathrm{i}: \gamma \hookrightarrow \Sigma$ which depend on fields only via their pull-back from $\Sigma$ to $\gamma$ :

$$
O_{\gamma, \mathrm{i}}(X)=O\left(\mathrm{i}^{*} X\right)
$$

where $X$ is the field. Such observables are automatically equivariant with respect to diffeomorphisms $\phi: \Sigma \rightarrow \Sigma$ in the sense that

$$
O_{\gamma, \phi \circ \mathrm{i}}\left(\left(\phi^{-1}\right)^{*} X\right)=O_{\gamma, \mathrm{i}}(X)
$$

where $\left(\phi^{-1}\right)^{*} X$ is the pull-back of the field by $\phi^{-1}$.

On a formal level, the expectation value of the observable

$$
\left\langle O_{\gamma, \mathrm{i}}\right\rangle=\int_{F_{\Sigma}} \mathcal{D} X O_{\gamma, \mathrm{i}}(X) \mathrm{e}^{i S_{\Sigma}(X)}
$$

is diffeomorphism-invariant

$$
\left\langle O_{\gamma, \phi \circ \mathrm{i}}\right\rangle=\left\langle O_{\gamma, \mathrm{i}}\right\rangle
$$

due to (2), diffeomorphism-invariance of the action $S_{\Sigma}$ and of the path integral measure $\mathcal{D} X$. This means in particular that the expectation value is an invariant of the embedding $\mathrm{i}: \gamma \hookrightarrow \Sigma$ under ambient isotopy.

Since topological field theories possess gauge symmetry, one also requires that the observable is gauge-invariant, so that in the path integral (3) one could pass to integration over gauge equivalence classes of fields. 
In this paper, we employ Batalin-Vilkovisky (BV) formalism to treat systems with gauge symmetry (cf., e.g., [18] for details). In particular, the space of fields $F_{\Sigma}$ becomes extended to an odd-symplectic $Q$-manifold $\mathcal{F}_{\Sigma}$ with the action $S_{\Sigma}$ extended to a function on $\mathcal{F}_{\Sigma}$, satisfying the master equation ${ }^{1}\left\{S_{\Sigma}, S_{\Sigma}\right\}=0$ and generating the cohomological vector field $Q_{\Sigma}$ as its Hamiltonian vector field. In this formalism, the gauge-invariance of the observable is expressed as

$$
Q_{\Sigma} O=0
$$

and the path integral (3) for the expectation value is traded for an integral over a Lagrangian submanifold $\mathcal{L}$ in $\mathcal{F}_{\Sigma}$, with $\mathcal{L}$ playing the role of the gauge-fixing condition.

One idea how to construct an observable for a gauge theory in BV formalism is to consider an extension of the BV theory $\left(\mathcal{F}_{\Sigma}, S_{\Sigma}\right)$ to a BV theory on a larger space $\left(\mathcal{F}_{\Sigma} \times \mathcal{F}^{\text {aux }}, S_{\Sigma}+S^{\text {aux }}\right)$ with $\mathcal{F}^{\text {aux }}$ the space of "auxiliary fields" and $S^{\text {aux }}$ the action for auxiliary fields $Y$ which is also allowed to depend on the "ambient" fields $X \in \mathcal{F}_{\Sigma}$. Then one uses the BV push-forward construction

$$
O(X)=\int_{\mathcal{L}^{\text {aux }} \subset \mathcal{F} \text { aux }} \mathcal{D} Y \mathrm{e}^{i S^{\text {aux }}(X, Y)}
$$

(Propositions 1, 3 in Section 4, cf. also [14]) to integrate out the auxiliary fields and produce an observable for the ambient theory $\left(\mathcal{F}_{\Sigma}, S_{\Sigma}\right)$. In this paper, we call such extensions by auxiliary fields "pre-observables" (to be more precise, we use a slightly better definition with Eq. (41) on $S^{\text {aux }}$ instead of the master equation on $S_{\Sigma}+S^{\text {aux }}$, which still suffices to produce an observable, cf. Definition 6 and Remark 5).

In this paper, we consider observables obtained by the construction outlined above for topological sigma models coming from AKSZ construction [1] (cf. a brief reminder in Section 2), where $\mathcal{F}_{\Sigma}$ is the mapping space from the tangent bundle of $\Sigma$ with shifted parity of fibers to a target $Q$-manifold $\mathcal{M}$ with additional Hamiltonian structure (cf. Definition 1). In this setting, we give a construction of pre-observables (Section 5), which associates a pre-observable for the AKSZ sigma model to an embedded submanifold i: $\gamma \hookrightarrow \Sigma$ and an extension of the target $\mathcal{M}$ to a $Q$-bundle over $\mathcal{M}$ with additional Hamiltonian structure in fibers (the "Hamiltonian $Q$-bundle", cf. Section 3). For the ambient and auxiliary actions to have degree zero, degrees of Hamiltonian structures in the base and the fiber of the target $Q$-bundle have to match ${ }^{2}$ dimensions of $\Sigma$ and $\gamma$, respectively.

\footnotetext{
${ }^{1}$ In this outline, we do not make explicit distinction between classical and quantum master equation. We write the classical one which also coincides with the quantum one if $\Delta_{\Sigma} S=0$ for $\Delta_{\Sigma}$ the BV Laplacian. Likewise, we write the classical gauge-invariance condition on the observable $Q_{\Sigma} O=0$ which coincides with the quantum one if $\Delta_{\Sigma} O=0$, cf. Section 4 for details.

${ }^{2}$ More precisely, degrees of Hamiltonians (not of symplectic structures) have to match the dimensions.
} 
Having a pre-observable for an AKSZ sigma model, we construct the corresponding observable by the BV push-forward (6). By construction, this observable is gauge-invariant in the sense of (5) and satisfies (1), and so formally produces expectation values which are invariant under ambient isotopy. Of course, the problem with this definition of the observable is that (6) is generally a path integral. In Section 6, we consider several simple cases when this integral can be made sense of and its expected properties can be rigorously checked:

(1) case when the fiber in the target is a point (which gives rise to observables given by an exponential of an integral of a local expression),

(2) case of one-dimensional observables (when the path integral becomes a path integral of quantum mechanical type and can be regularized via geometric quantization, which gives rise to certain generalization of Wilson loops),

(3) case when the integral (6) is Gaussian which gives rise to a class of observables which we call "torsion-like" for their similarity to Ray-Singer torsion.

One-dimensional observables (2) are similar to generalized Wilson loops constructed in $[6,15]$.

In Section 7, we give explicit examples of observables falling within one of the three classes above. In particular, we recover the usual Wilson loop observable in Chern-Simons theory, with the corresponding path integral expression being the Alekseev-Faddeev-Shatashvili path integral formula for the Wilson loop [2]. We also recover the Cattaneo-Rossi "Wilson surface" observable for $B F$ theory [10].

In this paper, we concentrate only on the construction of observables, we are not calculating their expectation values.

\subsection{LOGIC OF THE CONSTRUCTION}

(i) For an AKSZ sigma model on manifold $\Sigma$ with target $\mathcal{M}$, find a Hamiltonian $Q$-bundle $\mathcal{E}$ over $\mathcal{M}$. Then for every embedded submanifold $\mathrm{i}: \gamma \hookrightarrow \Sigma$ of fixed dimension matching the degree of Hamiltonian structure in fibers of $\mathcal{E}$, we construct a pre-observable.

(ii) Take the fiber BV integral (6) over the auxiliary fields of the pre-observable constructed in step (i) to obtain an observable for the AKSZ sigma model.

Unlike step (i), step (ii) is not canonical, in the sense that it is a quantization problem: the path integral has to be made sense of (and the gauge-invariance of the result has to be checked) which can be done for certain classes of target $Q$-bundles, but it is not clear whether it is possible to do in greater generality. ${ }^{3}$

\footnotetext{
${ }^{3}$ One can indeed try to define the path integral perturbatively, as sum of Feynman diagrams represented by integrals over compactified configuration spaces of tuples of points on $\gamma$. However, for the formal argument of Proposition 1 that the result is gauge-invariant to become rigorous, one has to prove that the hidden boundary strata of the configuration spaces appearing in the calculation of $Q_{\Sigma} O$ (cf. the proof of Proposition 6) do not contribute, which is not true in general case.
} 


\subsection{PLAN OF THE PAPER}

The logical organization of the exposition is as follows.

- Sections 2, 3.1, bits of 4 concerning BV observables-background reminders.

- Section 5-step (i) of the main construction.

- Section 3-auxiliary construction for step (i) of the main construction.

- Section 4-motivation for step (ii) of the main construction.

- Section 6- examples for step (ii) of the main construction.

- Section 7-fully explicit examples.

Section 2 is a short reminder of the AKSZ construction of topological sigma models in Batalin-Vilkovisky formalism. We also recall how some well-known topological field theories fit into the construction: Chern-Simons theory, $B F$ theory, Poisson sigma model.

In Section 3, we first briefly recall the standard notion of a $Q$-bundle and then define a "Hamiltonian" $Q$-bundle, preparing the grounds for the construction of pre-observables in Section 5. We start with the Definition 2 of a trivial Hamiltonian $Q$-bundle ("trivial" here means trivial as a fiber bundle; the cohomological vector field is not required to be a sum of a cohomological vector field on the base and another one on the fiber). All our examples are of this type, but for the completeness of the exposition, we also give a slightly more general Definition 3, which does not explicitly rely on the total space being a direct product. However, in a local trivialization Definition 3 boils down to Definition 2.

In Section 4, we recall the standard notions of classical and quantum observables in BV formalism and introduce the notion of a pre-observable, which comes in three modifications:

(i) Classical pre-observable, Definition 6: essentially, an extension of the action of the ambient classical BV theory to a solution of classical master equation on the space of fields extended by auxiliary fields. We give a technically more convenient definition with Eq. (41) required instead of the classical master equation on extended space of fields (cf. Remark 5 for the relation between the two).

(ii) Semi-quantum pre-observable, Definition 7, suited for integrating out auxiliary fields to obtain an observable for the ambient theory (Proposition 1).

(iii) Quantum pre-observable for a quantum ambient BV theory (i.e., one with the action satisfying the quantum master equation), Definition 9: this is also an extension of the ambient theory by auxiliary fields, plus an extension of the action to a solution of quantum master equation on the extended space. From a quantum pre-observable, one can induce a quantum observable for the ambient theory, by integrating out auxiliary fields (Proposition 3).

In this section, in the discussion of quantization, we always work with spaces of fields as with finite-dimensional spaces and the integrals are over finite-dimensional super-manifolds. In the context of local field theory, the BV push-forward becomes 
a path integral, so the proofs given in Section 4 stop to work and the propositions become conjectures that have to be proven by more delicate means (cf. the proofs of Propositions 5, 6 in Section 6).

Section 5 is the logical core of the paper. Here, we give the construction of a classical pre-observable for an AKSZ theory out of an extension of the target to a Hamiltonian $Q$-bundle.

In Section 6, we treat several classes of situations when the BV push-forward yielding an observable out of a pre-observable constructed in Section 5 can be performed rigorously: case of target fiber being a point, case of one-dimensional observables (via geometric quantization), case when the BV push-forward is given by a Gaussian integral.

In Section 7, we specialize the constructions of Section 6 to present several explicit examples, including the Wilson loop together with its path integral representation known from [2], Cattaneo-Rossi codimension 2 observable for $B F$ theory, torsion observables in Chern-Simons and $B F$ theories, etc.

\section{Terminology, Notations}

\section{Terminology.}

- $Q$-manifold (or bundle) $=$ differential graded manifold (or bundle).

- Ghost number = internal degree (to distinguish from de Rham degree of a differential form) $=\mathbb{Z}$-grading on functions on $\mathbb{Z}$-graded manifolds.

- Observable $=$ gauge-invariant functional on the space of fields (cf. Section 4 for definitions).

- Expectation value $=$ correlator

Conventions. We set the Planck's constant $\hbar=1$ (cf. Remark 6 on how to reintroduce $\hbar$ ).

Notations. We use $\mathcal{M}, \mathcal{N}, \mathcal{E}, \mathcal{F}$, etc., for $\mathbb{Z}$-graded manifolds, $M, N$, etc., for ordinary (non-graded) manifolds; $\Sigma$ always denotes the source (spacetime) manifold of the sigma model, $\gamma$ is typically a submanifold of $\Sigma$ on which the observable is supported. We use $\mathcal{L}$ for a Lagrangian submanifold of a degree -1 symplectic graded manifold.

De Rham differential on the target of the sigma model and on the space of fields is denoted $\delta$, whereas $d$ is reserved for the de Rham differential on the source manifold.

We denote by $\mathfrak{X}(\mathcal{M})$ the Lie algebra of vector fields on $\mathcal{M}$. For a fiber bundle $\pi: \mathcal{E} \rightarrow \mathcal{M}$, we denote by $\mathfrak{X}^{\text {vert }}(\mathcal{E})$ the Lie algebra of vertical vector fields on the total space $\mathcal{E}$, i.e., the space of sections $\Gamma\left(\mathcal{E}, T^{\text {vert }} \mathcal{E}\right)$ of the vertical distribution on $\mathcal{E}, T^{\text {vert }} \mathcal{E}=\operatorname{ker}(d \pi) \subset T \mathcal{E}$.

In a Hamiltonian $Q$-bundle (see Definitions 2, 3 in Section 3), $Q$ stands both for the cohomological vector field on the base and for its horizontal lift to the total space. 
We denote the degree (the ghost number) of functions/differential forms/vector fields on a graded manifold by $|\cdots|$.

\section{AKSZ Reminder}

What follows is a very short reminder of the AKSZ construction of topological sigma models in Batalin-Vilkovisky formalism, to fix the terminology and notation. We refer the reader to the original paper [1] and the later expositions in $[8,16]$ for details.

\subsection{TARGET DATA}

Let $\mathcal{M}$ be a degree $n$ symplectic $Q$-manifold, i.e., a $\mathbb{Z}$-graded manifold endowed with a degree 1 vector field $Q$ satisfying $Q^{2}=0$ (the cohomological vector field) and with a degree ${ }^{4} n$ symplectic form $\omega \in \Omega^{2}(\mathcal{M})$ which is compatible with $Q$, i.e., $L_{Q} \omega=0$.

Assume $^{5}$ that $Q$ has a Hamiltonian function $\Theta \in C^{\infty}(\mathcal{M})$ with $|\Theta|=n+1$, $\{\Theta, \bullet\}_{\omega}=Q$ and satisfying

$$
\{\Theta, \Theta\}_{\omega}=0
$$

Also assume that $\omega$ is exact, with $\alpha \in \Omega^{1}(\mathcal{M})$ a primitive.

DEFINITION 1. We call the set of data $(\mathcal{M}, Q, \omega=\delta \alpha, \Theta)$ a Hamiltonian $Q$ manifold of degree $n$.

\subsection{THE AKSZ SIGMA MODEL}

Fix a Hamiltonian $Q$-manifold $\mathcal{M}$ of degree $n \geq-1$ and let $\Sigma$ be an oriented closed manifold, $\operatorname{dim} \Sigma=n+1$. Then one constructs the space of fields as the space of graded maps between graded manifolds from the degree-shifted tangent bundle $T[1] \Sigma$ to $\mathcal{M}$ :

$$
\mathcal{F}_{\Sigma}=\operatorname{Map}(T[1] \Sigma, \mathcal{M})
$$

It is a $Q$-manifold with the cohomological vector field coming from the lifting of $Q$ on the target and of the de Rham operator $d_{\Sigma}$ on $\Sigma$ (viewed as a cohomological vector field on $T[1] \Sigma$ ) to the mapping space:

$$
Q_{\Sigma}=\left(d_{\Sigma}\right)^{\text {lifted }}+(Q)^{\text {lifted }} \in \mathfrak{X}\left(\mathcal{F}_{\Sigma}\right)
$$

\footnotetext{
${ }^{4}$ By "degree" here we mean the internal $\mathbb{Z}$-grading coming from the grading on $\mathcal{M}$.

${ }^{5}$ In fact (cf. [16]), for $n \neq-1$ the symplectic property of the cohomological vector field $\left(L_{Q} \omega=0\right)$ implies existence and uniqueness of the Hamiltonian $\Theta=\frac{1}{n+1} \iota^{\iota} \iota Q^{\omega}$ where $E$ is the Euler vector field. Maurer-Cartan equation (7) follows from $Q^{2}=0$ for $n \neq-2$. Also, for $n \neq 0$, a closed form $\omega$ is automatically exact.
} 
Transgression map. The following natural maps

$$
\begin{gathered}
\mathcal{F}_{\Sigma} \times T[1] \Sigma \stackrel{\text { ev }}{\longrightarrow} \mathcal{M} \\
\quad p \downarrow \\
\quad \mathcal{F}_{\Sigma}
\end{gathered}
$$

(where $p$ is the projection to the first factor) allow us to define the transgression map

$$
\tau_{\Sigma}=p_{*} \mathrm{ev}^{*}: \quad \Omega^{\bullet}(\mathcal{M}) \rightarrow \Omega^{\bullet}\left(\mathcal{F}_{\Sigma}\right)
$$

Here, $p_{*}$ is the fiber integration over $T[1] \Sigma$ (with the canonical integration measure). Map $\tau_{\Sigma}$ preserves the de Rham degree of a form, but changes the internal grading (the ghost number) by $-\operatorname{dim} \Sigma$.

If $u \in \mathfrak{X}(T[1] \Sigma), v \in \mathfrak{X}(\mathcal{M})$ are any vector fields on the source and the target and $\psi \in \Omega^{\bullet}(\mathcal{M})$ is a form on the target, then for the Lie derivatives of the transgressed form along the lifted vector fields we have

$$
\begin{aligned}
& L_{u \text { lifted }} \tau_{\Sigma}(\psi)=0 \\
& L_{v^{\text {lifted }} \tau_{\Sigma}(\psi)}=(-1)^{|v| \operatorname{dim} \Sigma} \tau_{\Sigma}\left(L_{v} \psi\right)
\end{aligned}
$$

The finite version of (12) is that for $\Phi: T[1] \Sigma \rightarrow T[1] \Sigma$ a diffeomorphism of the source, we have

$$
\left(\Phi^{*}\right)^{*} \tau_{\Sigma}(\psi)=\tau_{\Sigma}(\psi)
$$

where $\Phi^{*}: \mathcal{F}_{\Sigma} \rightarrow \mathcal{F}_{\Sigma}$ is the lifting of $\Phi$ to the mapping space and $\left(\Phi^{*}\right)^{*}: \Omega^{\bullet}\left(\mathcal{F}_{\Sigma}\right) \rightarrow$ $\Omega^{\bullet}\left(\mathcal{F}_{\Sigma}\right)$ is the pull-back by $\Phi^{*}$.

The BV 2-form and the master action. One obtains the degree -1 symplectic form (the "BV 2-form") on $\mathcal{F}_{\Sigma}$ from the target by transgression"

$$
\Omega_{\Sigma}=(-1)^{\operatorname{dim} \Sigma} \tau_{\Sigma}(\omega) \quad \in \Omega^{2}\left(\mathcal{F}_{\Sigma}\right)
$$

The Hamiltonian function for $Q_{\Sigma}$ (the master action) is constructed as

$$
S_{\Sigma}=\underbrace{\imath}_{S_{\Sigma}^{\text {kin }}} \underbrace{\tau_{\Sigma}(\Theta)}_{d_{\Sigma}^{\text {lifted }} \tau_{\Sigma}(\alpha)} \in C^{\infty}\left(\mathcal{F}_{\Sigma}\right)
$$

It automatically satisfies the classical master equation

$$
\left\{S_{\Sigma}, S_{\Sigma}\right\}_{\Omega_{\Sigma}}=0
$$

\footnotetext{
${ }^{6}$ We introduce the sign $(-1)^{\operatorname{dim} \Sigma}$ in this definition to avoid signs in the formula for the action below. The reader may encounter different sign conventions for the AKSZ construction in the literature.
} 
One can summarize the construction above by saying that we have a degree -1 Hamiltonian $Q$-manifold structure on the mapping space (8): $\left(\mathcal{F}_{\Sigma}, Q_{\Sigma}, \Omega_{\Sigma}, S_{\Sigma}\right)$. The primitive 1-form for the BV 2-form can also be constructed by transgression as $\tau_{\Sigma}(\alpha)$.

Note that exact shifts of the target 1-form, $\alpha \mapsto \alpha+\delta f$, with $f \in C^{\infty}(M)$ leave $S_{\Sigma}$ unchanged. $^{7}$

Why AKSZ theory is topological. For $\phi \in \operatorname{Diff}(\Sigma)$ a diffeomorphism of $\Sigma$, denote $\tilde{\phi} \in \operatorname{Diff}(T[1] \Sigma)$ the tangent lift of $\phi$ to $T[1] \Sigma$. Then

$$
\left(\tilde{\phi}^{*}\right)^{*} S_{\Sigma}=S_{\Sigma}, \quad\left(\tilde{\phi}^{*}\right)^{*} \Omega_{\Sigma}=\Omega_{\Sigma}
$$

because of (14) and because $d_{\Sigma} \in \mathfrak{X}(T[1] \Sigma)$ commutes with $\tilde{\phi}$, since the latter is a tangent lift.

In coordinates. Let $x^{a}$ be local homogeneous coordinates on the target $\mathcal{M}$, let $u^{\mu}$ be local coordinates on $\Sigma$ and $\theta^{\mu}=d u^{\mu}$ be the associated degree 1 fiber coordinates on $T[1] \Sigma$. Then, locally an element of $\mathcal{F}_{\Sigma}$ is parameterized by

$$
X^{a}(u, \theta)=\sum_{k=0}^{\operatorname{dim} \Sigma} \underbrace{\sum_{1 \leq \mu_{1}<\cdots<\mu_{k} \leq \operatorname{dim} \Sigma} X_{\mu_{1} \cdots \mu_{k}}^{a}(u) \theta^{\mu_{1}} \cdots \theta^{\mu_{k}}}_{X_{(k)}^{a}(u, \theta)}
$$

Coefficient functions $X_{\mu_{1} \cdots \mu_{k}}^{a}(u)$ are local coordinates of degree $\left|x^{a}\right|-k$ on the mapping space $\mathcal{F}_{\Sigma}$. Expression (18) is known as (the component of) the superfield, and it can be regarded as a generating function for the coordinates on the mapping space $\mathcal{F}_{\Sigma}$.

For any function $f \in C^{\infty}(\mathcal{M})$, we have

$$
\begin{aligned}
\operatorname{ev}^{*} f=f(X)= & f\left(X_{(0)}\right)+X_{\geq 1}^{a} \cdot\left(\partial_{a} f\right)\left(X_{(0)}\right)+\frac{1}{2} X_{\geq 1}^{a} X_{\geq 1}^{b} \cdot\left(\partial_{b} \partial_{a} f\right)\left(X_{(0)}\right)+\cdots \\
& \in C^{\infty}\left(\mathcal{F}_{\Sigma} \times T[1] \Sigma\right)
\end{aligned}
$$

where we denoted $X_{\geq 1}^{a}=\sum_{k \geq 1} X_{(k)}^{a}$ the part of the superfield of positive de Rham degree with respect to $\Sigma$; ev is the horizontal arrow in (10).

Let $\alpha$ and $\omega$ be locally given as $\alpha=\alpha_{a}(x) \delta x^{a}$ and $\omega=\frac{1}{2} \omega_{a b}(x) \delta x^{a} \wedge \delta x^{b}$. Then the BV 2-form and its primitive are:

$$
\Omega_{\Sigma}=(-1)^{\operatorname{dim} \Sigma} \int_{\Sigma} \frac{1}{2} \omega_{a b}(X) \delta X^{a} \wedge \delta X^{b}, \quad \alpha_{\Sigma}=\int_{\Sigma} \alpha_{a}(X) \delta X^{a}
$$

(Note that we use $\delta$ to denote the de Rham differential on the target $\mathcal{M}$ and on the mapping space $\mathcal{F}_{\Sigma}$. We reserve symbol $d$ for the de Rham differential on the source $\Sigma$.)

\footnotetext{
${ }^{7}$ Indeed, under such a shift we have $\tau_{\Sigma}(\alpha) \mapsto \tau_{\Sigma}(\alpha)+p_{*} \delta \operatorname{ev}^{*}(f)$, and hence $S_{\Sigma} \mapsto S_{\Sigma}+$ $\int_{\Sigma} d_{\Sigma} \mathrm{ev}^{*} f$ and the last term vanishes by Stokes' theorem.
} 
The master action is:

$$
S_{\Sigma}(X)=\underbrace{\int_{\Sigma} \alpha_{a}(X) \mathrm{d} X^{a}}_{S_{\Sigma}^{\text {kin }}}+\underbrace{\int_{\Sigma} \Theta(X)}_{S_{\Sigma}^{\text {target }}}
$$

If the cohomological vector field on the target is locally written as $Q=Q^{a}(x) \frac{\partial}{\partial x^{a}}$ then the cohomological vector field (9) is determined by its action on the components of the superfield:

$$
Q_{\Sigma} X^{a}=d X^{a}+Q^{a}(X)
$$

Critical points of $S_{\Sigma}$ are (with our sign conventions) $Q$-anti-morphisms between $T[1] \Sigma$ and $\mathcal{M}$, i.e., $Q$-morphisms between $(T[1] \Sigma, d)$ and $(\mathcal{M},-Q)$.

\subsection{EXAMPLES}

Here, we recall some of the standard examples of the AKSZ construction.

Chern-Simons theory [1]. Let $\mathfrak{g}$ be a quadratic Lie algebra, i.e., a Lie algebra with a non-degenerate invariant pairing (,). Denote by $\psi: \mathfrak{g}[1] \rightarrow \mathfrak{g}$ the degree $1 \mathfrak{g}$-valued coordinate on $\mathfrak{g}[1]$. We choose the target Hamiltonian $Q$-manifold of degree 2 as

$$
\begin{aligned}
\mathcal{M} & =\mathfrak{g}[1], \quad Q=\left\langle\frac{1}{2}[\psi, \psi], \frac{\partial}{\partial \psi}\right\rangle, \\
\omega & =\frac{1}{2}(\delta \psi, \delta \psi), \quad \alpha=\frac{1}{2}(\psi, \delta \psi), \quad \Theta=\frac{1}{6}(\psi,[\psi, \psi])
\end{aligned}
$$

where $\langle$,$\rangle is the canonical pairing { }^{8}$ between $\mathfrak{g}$ and $\mathfrak{g}^{*}$. The associated AKSZ sigma model on a closed oriented 3-manifold $\Sigma$ has the space of fields

$$
\mathcal{F}_{\Sigma}=\operatorname{Map}(T[1] \Sigma, \mathfrak{g}[1]) \cong \mathfrak{g}[1] \otimes \Omega^{\bullet}(\Sigma)
$$

The superfield is

$$
A=A_{(0)}+A_{(1)}+A_{(2)}+A_{(3)}
$$

with $A_{(k)}$ a coordinate on $\mathcal{F}_{\Sigma}$ with values in $\mathfrak{g}$-valued $k$-forms on $\Sigma$, with internal degree (ghost number) $1-k$, for $k=0,1,2,3$. The BV 2 -form is

$$
\Omega_{\Sigma}=-\frac{1}{2} \int_{\Sigma}(\delta A, \delta A)
$$

and the action is

$$
S_{\Sigma}=\int_{\Sigma} \frac{1}{2}(A, d A)+\frac{1}{6}(A,[A, A])
$$

${ }^{8}$ We will generally be using notation $\langle$,$\rangle for the canonical pairing V \otimes V^{*} \rightarrow \mathbb{R}$ between a vector space $V$ and its dual; sometimes we will indicate the respective $V$ as a subscript: $\langle,\rangle_{V}$. 
This is the action of Chern-Simons theory in Batalin-Vilkovisky formalism.

In case $\mathfrak{g}=\mathbb{R}$ with abelian Lie algebra structure, we have $Q=\Theta=0$ on the target and

$$
\mathcal{F}_{\Sigma}=\Omega^{\bullet}(\Sigma)[1], \quad \Omega_{\Sigma}=-\frac{1}{2} \int_{\Sigma} \delta A \wedge \delta A, \quad S_{\Sigma}=\frac{1}{2} \int_{\Sigma} A \wedge d A
$$

This is the abelian Chern-Simons theory in BV formalism.

$B F$ theory. For $\mathfrak{g}$ a Lie algebra (not necessarily quadratic ${ }^{9}$ ) and $D$ a nonnegative integer, we define the target Hamiltonian $Q$-manifold of degree $D-1$ as

$$
\begin{aligned}
\mathcal{M} & =\mathfrak{g}[1] \oplus \mathfrak{g}^{*}[D-2], \quad Q=\left\langle\frac{1}{2}[\psi, \psi], \frac{\partial}{\partial \psi}\right\rangle+\left\langle\operatorname{ad}_{\psi}^{*} \xi, \frac{\partial}{\partial \xi}\right\rangle, \\
\omega & =\langle\delta \xi, \delta \psi\rangle, \quad \alpha=\langle\xi, \delta \psi\rangle, \quad \Theta=\frac{1}{2}\langle\xi,[\psi, \psi]\rangle
\end{aligned}
$$

Here, $\psi: \mathfrak{g}[1] \rightarrow \mathfrak{g}$ is as before and $\xi: \mathfrak{g}^{*}[D-2] \rightarrow \mathfrak{g}^{*}$ is the $\mathfrak{g}^{*}$-valued coordinate on $\mathfrak{g}^{*}[D-2]$ of degree $D-2$; ad $\mathfrak{d}^{*}$ is the coadjoint action of $\mathfrak{g}$ on $\mathfrak{g}^{*}$.

The associated AKSZ sigma model on a closed oriented $D$-manifold $\Sigma$ has the space of fields

$$
\mathcal{F}_{\Sigma}=\mathfrak{g}[1] \otimes \Omega^{\bullet}(\Sigma) \oplus \mathfrak{g}^{*}[D-2] \otimes \Omega^{\bullet}(\Sigma)
$$

The superfields associated to $\psi$ and $\xi$ are, respectively,

$$
A=\sum_{k=0}^{D} A_{(k)}, \quad B=\sum_{k=0}^{D} B_{(k)}
$$

with $A_{(k)}$ a $\mathfrak{g}$-valued $k$-form on $\Sigma$ of internal degree $1-k ; B_{(k)}$ is a $\mathfrak{g}^{*}$-valued $k$ form of internal degree $D-2-k$. The $\mathrm{BV} 2$-form and the action are:

$$
\Omega_{\Sigma}=(-1)^{D} \int_{\Sigma}\langle\delta B, \delta A\rangle, \quad S_{\Sigma}=\int_{\Sigma}\left\langle B, d A+\frac{1}{2}[A, A]\right\rangle
$$

This is the $B F$ theory in BV formalism.

In abelian case, $\mathfrak{g}=\mathbb{R}$, we have $Q=\Theta=0$ on the target and

$$
\begin{aligned}
& \mathcal{F}_{\Sigma}=\Omega^{\bullet}(\Sigma)[1] \oplus \Omega^{\bullet}(\Sigma)[D-2], \\
& \Omega_{\Sigma}=(-1)^{D} \int_{\Sigma} \delta B \wedge \delta A, \quad S_{\Sigma}=\int_{\Sigma} B \wedge d A
\end{aligned}
$$

Poisson sigma model [8]. Let $M$ be a manifold endowed with a Poisson bivector $\pi \in \Gamma\left(M, \wedge^{2} T M\right)$. We construct the target Hamiltonian $Q$-manifold of degree 1 as

$$
\begin{aligned}
\mathcal{M} & =T^{*}[1] M, \\
Q & =\left\langle\pi(x), p \wedge \frac{\partial}{\partial x}\right\rangle_{\wedge^{2} T_{x} M}+\frac{1}{2}\left\langle\frac{\partial}{\partial x} \pi(x),(p \wedge p) \otimes \frac{\partial}{\partial p}\right\rangle_{\left(\wedge^{2} T \otimes T^{*}\right)_{x} M}, \\
\omega & =\langle\delta p, \delta x\rangle, \quad \alpha=\langle p, \delta x\rangle, \quad \Theta=\frac{1}{2}\langle\pi(x), p \wedge p\rangle_{\wedge^{2} T_{x} M}
\end{aligned}
$$

${ }^{9}$ However, for the consistency of quantization, in particular for the quantum master equation (49), one has to require that $\mathfrak{g}$ is unimodular. 
Here, $x$ and $p$ stand for the local base and fiber coordinates on $T^{*}[1] M$, respectively. Note that all objects in (27) are globally well defined.

The corresponding AKSZ sigma model on an oriented closed surface $\Sigma$ has the space of fields

$$
\mathcal{F}_{\Sigma}=\operatorname{Map}\left(T[1] \Sigma, T^{*}[1] M\right)
$$

with superfields

$$
X=X_{(0)}+X_{(1)}+X_{(2)}, \quad \eta=\eta_{(0)}+\eta_{(1)}+\eta_{(2)}
$$

associated to local coordinates $x$ and $p$ on the target, respectively. Here, $X_{(k)}$ and $\eta_{(k)}$ are $k$-forms on $\Sigma$ with internal degrees $-k$ and $1-k$, respectively. The BV 2form and the action are:

$$
\Omega_{\Sigma}=\int_{\Sigma}\langle\delta \eta, \delta X\rangle, \quad S_{\Sigma}=\int_{\Sigma}\langle\eta, \mathrm{d} X\rangle+\frac{1}{2}\langle\pi(X), \eta \wedge \eta\rangle
$$

\section{Hamiltonian $Q$-Bundles}

In this section, we briefly recall the standard notion of a $Q$-bundle and then introduce "Hamiltonian $Q$-bundles", an auxiliary notion necessary for our construction of observables.

\section{1. $Q$-BUNDLES REMINDER}

Recall that a " $Q$-bundle" $[19,12]$ is a fiber bundle in the category of $Q$-manifolds.

In particular, a trivial $Q$-bundle is trivial bundle of graded manifolds

$$
\pi: \underbrace{\mathcal{M} \times \mathcal{N}}_{\mathcal{E}} \rightarrow \mathcal{M}
$$

where the base $\mathcal{M}$ is endowed with a cohomological vector field $Q$ and the total space is endowed with a cohomological vector field $Q^{\text {tot }}$ in such a way that $\pi$ is a $Q$-morphism, i.e., $d \pi\left(Q^{\text {tot }}\right)=Q$. This implies the following ansatz for $Q^{\text {tot }}$ :

$$
Q^{\text {tot }}=Q+\mathcal{A}
$$

where $\mathcal{A} \in \mathfrak{X}^{\text {vert }}(\mathcal{E}) \cong \mathfrak{X}(\mathcal{N}) \hat{\otimes} C^{\infty}(\mathcal{M})$ is the vertical part of $Q^{\text {tot }}$. Cohomological property for $Q^{\text {tot }}$ is equivalent to

$$
Q \mathcal{A}+\frac{1}{2}[\mathcal{A}, \mathcal{A}]=0
$$

plus the cohomological property for $Q$. Note that in the first term on the 1.h.s. of (29) we are thinking of $Q$ as acting on $C^{\infty}(\mathcal{M})$ part of $\mathcal{A}$. Equivalently, we can lift $Q$ to a horizontal vector field on $\mathcal{E}$ and write the first term as $[Q, \mathcal{A}]$. 


\subsection{TRIVIAL HAMILTONIAN $Q$-BUNDLES}

DEFINITION 2. We define a trivial Hamiltonian $Q$-bundle of degree $n^{\prime} \in \mathbb{Z}$ as the following collection of data.

(i) A trivial $Q$-bundle

$$
\pi: \mathcal{E}=\mathcal{M} \times \mathcal{N} \rightarrow \mathcal{M}
$$

with $Q^{\text {tot }}=Q+\mathcal{A}$ as in (28).

(ii) The fiber $\mathcal{N}$ is endowed with a degree $n^{\prime}$ exact symplectic form $\omega^{\prime}=\delta \alpha^{\prime}$ and a degree $\left(n^{\prime}+1\right)$ Hamiltonian $\Theta^{\prime} \in C^{\infty}(\mathcal{E})$ satisfying $\mathcal{A}=\left\{\Theta^{\prime}, \bullet\right\}_{\omega^{\prime}}$ and

$$
Q \Theta^{\prime}+\frac{1}{2}\left\{\Theta^{\prime}, \Theta^{\prime}\right\}_{\omega^{\prime}}=0
$$

Remark 1. (Roytenberg) Symplectic structure $\omega^{\prime}$ is automatically exact for $n^{\prime} \neq 0$, since $\omega^{\prime}=\delta\left(\frac{1}{n^{\prime}} \iota \omega^{\prime}\right)$ where $E$ is the Euler vector field on $\mathcal{N}$.

Remark 2. If $n^{\prime}=0$, then $\omega^{\prime}$ is not automatically exact. In this situation, exactness condition may be relaxed ${ }^{10}$ to the Bohr-Sommerfeld condition that $\omega^{\prime} / 2 \pi$ defines an integral cohomology class in $H^{2}(\mathcal{N})$.

Then the role of primitive 1-form $\alpha^{\prime}$ is taken by a Hermitian line bundle $L^{\prime}$ over $\mathcal{N}$ equipped with a $U(1)$-connection $\nabla^{\prime}$ of curvature $\omega^{\prime}$.

Remark 3. For a Hamiltonian $Q$-manifold of degree $n \neq-2$, equation $\{\Theta, \Theta\}=0$ follows from the fact that $\Theta$ is the Hamiltonian for a cohomological vector field (Jacobi identity implies $\left\{\frac{1}{2}\{\Theta, \Theta\}, \bullet\right\}=Q^{2}(\bullet)=0$ which implies that $\{\Theta, \Theta\}$ is a constant of degree $n+2$ and thus vanishes unless $n=-2$ ).

Observe that this argument fails for the fiber $\mathcal{N}$ : applying the same reasoning to derive the Eq. (30) from (29), we obtain that 1.h.s. of (30) is a pull-back of some function on the base of degree $n^{\prime}+2$, which does not imply that it is zero.

\subsection{GENERAL HAMILTONIAN $Q$-BUNDLES}

The assumption of triviality of the bundle $\mathcal{E}$, used in the Definition 2, can be relaxed as follows.

DEFINITION 3. A Hamiltonian $Q$-bundle is the following set of data:

(i) A $Q$-bundle $\pi: \mathcal{E} \rightarrow \mathcal{M}$.

(ii) A degree $n^{\prime}$ exact pre-symplectic form $\omega^{\prime}=\delta \alpha^{\prime}$ on the total space $\mathcal{E}$, with the property that the distribution $\operatorname{ker} \omega^{\prime} \subset T \mathcal{E}$ is transversal to the vertical distribution $T^{\text {vert }} \mathcal{E}$; thus $\operatorname{ker} \omega^{\prime}$ defines a flat Ehresmann connection $\nabla_{\omega^{\prime}}$ on $\mathcal{E}$.

\footnotetext{
${ }^{10} \mathrm{Cf}$. Remark 8 for the motivation: primitive $\alpha^{\prime}$ (or connection $\nabla^{\prime}$ in the relaxed version) will be needed in the construction of Section 5 to define the kinetic part of the auxiliary action.
} 
(iii) A Hamiltonian function $\Theta^{\prime} \in C^{\infty}(\mathcal{E})$ satisfying

$$
\iota^{\mathrm{tot}} \omega^{\prime}=\delta^{\mathrm{vert}} \Theta^{\prime}
$$

where $\delta^{\text {vert }}=\left(i^{\text {vert }}\right)^{*}$ o $\delta$ is the vertical part of de Rham differential on $\mathcal{E}$; we denoted $i^{\text {vert }}: T^{\text {vert }} \mathcal{E} \hookrightarrow T \mathcal{E}$ the natural inclusion. We also require that

$$
\left(Q^{\text {hor }}+\frac{1}{2} Q^{\text {vert }}\right) \Theta^{\prime}=0
$$

where the splitting $Q^{\text {tot }}=Q^{\text {hor }}+Q^{\text {vert }}$ of the cohomological vector field on $\mathcal{E}$ into the horizontal and the vertical part is defined by connection $\nabla_{\omega^{\prime}}$.

Remark 4. Note that one can introduce a local trivialization of $\mathcal{E}$ consistent with the flat connection $\nabla_{\omega^{\prime}}$ (i.e., where the horizontal distribution $\operatorname{ker} \omega^{\prime} \subset T \mathcal{E}$ is defined by the direct product structure on $\left.\mathcal{E}\right|_{U}$ coming from the local trivialization, where $U \subset \mathcal{M}$ is a trivializing neighborhood). In such a trivialization, we recover back the Definition 2. ${ }^{11}$ In this sense, Definition 3 does not bring in a drastic increase of generality.

\subsection{EXAMPLES}

(i) Let $\mathfrak{g}$ be a Lie algebra and let $\rho: \mathfrak{g} \rightarrow \mathfrak{s o}(R) \subset \operatorname{End}(R)$ be a representation of $\mathfrak{g}$ on by anti-symmetric matrices on a Euclidean vector space $R,($,$) . Then for$ any $k \in \mathbb{Z}$ we can define a degree $4 k+2$ trivial Hamiltonian $Q$-bundle with base $\mathcal{M}=\mathfrak{g}[1]$ and fiber $\mathcal{N}=R[2 k+1]$. Let $\psi$ be the degree $1 \mathfrak{g}$-valued coordinate on $\mathfrak{g}[1]$ and let $x$ be the degree $2 k+1 R$-valued coordinate on $R[2 k+1]$. Then, we set

$$
Q=\frac{1}{2}\left\langle[\psi, \psi], \frac{\partial}{\partial \psi}\right\rangle
$$

- the Chevalley-Eilenberg differential, and

$$
\begin{aligned}
\mathcal{A} & =\left\langle\rho(\psi) x, \frac{\partial}{\partial x}\right\rangle, \quad \omega^{\prime}=\frac{1}{2}(\delta x, \delta x), \\
\alpha^{\prime} & =\frac{1}{2}(x, \delta x), \quad \Theta^{\prime}=\frac{1}{2}(x, \rho(\psi) x)
\end{aligned}
$$

where $\langle$,$\rangle denotes the canonical pairing between a vector space and its dual.$ It is a straightforward check that this comprises the data of a trivial Hamiltonian $Q$-bundle of degree $4 k+2$, according to Definition 2 .

As a variation of this example, we may require that the pairing (, ) is antisymmetric (so that $R$ is a symplectic space instead of Euclidean) and that

${ }^{11}$ In particular, Eq. (31) corresponds to (30), since one can write the latter as $\left(Q+\frac{1}{2} \mathcal{A}\right) \Theta^{\prime}=0$ and assign $Q=Q^{\text {hor }}, \mathcal{A}=Q^{\text {vert }}$. 
$\rho: \mathfrak{g} \rightarrow \mathfrak{s p}(R) \subset \operatorname{End}(R)$ is a symplectic representation. Then formulae $(32,33)$ define a Hamiltonian $Q$-bundle structure of degree $4 k$ on $\mathfrak{g}[1] \times R[2 k]$ (note that here we choose an even degree shift for the fiber).

(ii) Example above generalizes straightforwardly to the setting of $L_{\infty}$ algebras and $L_{\infty}$ modules. Namely, let $\mathfrak{g}$ be an $L_{\infty}$ algebra with operations $\left\{l_{j}: \wedge^{j} \mathfrak{g} \rightarrow\right.$ $\mathfrak{g}\}_{j \geq 1}$. Also, let $R$ be graded vector space with graded symmetric pairing (,) of degree $q$ and with the structure of an $L_{\infty}$ module over $\mathfrak{g}$ with the module operations $\left\{\rho_{j}: \wedge^{j} \mathfrak{g} \otimes R \rightarrow R\right\}_{j \geq 0}$, where we additionally require that operations $\left(\bullet, \rho_{j}(\cdots ; \bullet)\right)$ are graded anti-symmetric w.r.t. the two entries in the module. For $\psi$, a $\mathfrak{g}$-valued degree 1 coordinate on $\mathfrak{g}[1]$ and $x$ a $R$-valued degree $2 k+1$ coordinate on $R[2 k+1]$, we construct a trivial Hamiltonian $Q$ bundle with $\mathcal{M}=\mathfrak{g}[1], \mathcal{N}=R[2 k+1]$ and the data are:

$$
Q=\sum_{j \geq 1} \frac{1}{j !}\left\langle l_{j}(\psi, \ldots, \psi), \frac{\partial}{\partial \psi}\right\rangle
$$

for the base and

$$
\begin{aligned}
\mathcal{A} & =\sum_{j \geq 0} \frac{1}{j !}\left\langle\rho_{j}(\psi, \ldots, \psi ; x), \frac{\partial}{\partial x}\right\rangle, \omega^{\prime}=\frac{1}{2}(\delta x, \delta x), \\
\alpha^{\prime} & =\frac{1}{2}(x, \delta x), \Theta^{\prime}=\sum_{j \geq 0} \frac{1}{2 j !}\left(x, \rho_{j}(\psi, \ldots, \psi ; x)\right)
\end{aligned}
$$

This comprises the data of a trivial Hamiltonian $Q$-bundle of degree $4 k+2+$ $q$.

(iii) Example (ii) admits the following variation. If $\mathfrak{h}$ is an $L_{\infty}$ algebra with $I \subset \mathfrak{h}$ an $L_{\infty}$ ideal, ${ }^{12}$ then there is a natural $Q$-bundle

$$
\pi: \mathfrak{h}[1] \rightarrow(\mathfrak{h} / I)[1]
$$

with bundle projection being the quotient map and with the fiber $I$. If in addition we fix a section of the quotient map $\mathfrak{h} \rightarrow \mathfrak{h} / I$, i.e., a splitting of the short exact sequence

$$
I \rightarrow \mathfrak{h} \rightarrow \mathfrak{h} / I
$$

and if $I$ carries a degree $q$ cyclic $^{13}$ inner product, then (36) becomes a Hamiltonian $Q$-bundle of degree $2+q$. Explicitly, the structure on the base $\mathcal{M}=$ $(\mathfrak{h} / I)[1]$ is again given by (34) with $\left\{l_{j}\right\}$ the quotient $L_{\infty}$ operations on $\mathfrak{h} / I$,

\footnotetext{
${ }^{12}$ Recall that a subspace $I \subset \mathfrak{h}$ is called an $L_{\infty}$ ideal if the $L_{\infty}$ operations on $\mathfrak{h}$ take values in $I$ if at least one argument is in $I$.

${ }^{13}$ By cyclic property for the pairing on the ideal we mean that extending the pairing on $I$ to $\mathfrak{h}$ by zero on $\mathfrak{h} / I$, we obtain a degenerate cyclic pairing for the $L_{\infty}$ algebra $\mathfrak{h}$.
} 
$\psi$ the degree $1(\mathfrak{h} / I)$-valued coordinate on $(\mathfrak{h} / I)[1]$. The structure on the fiber $\mathcal{N}=I[1]$ is:

$$
\begin{aligned}
\mathcal{A} & =\sum_{j \geq 0, k \geq 0, j+k \geq 1} \frac{1}{j ! k !}\left\langle P_{I} \lambda_{j+k}(\underbrace{\psi, \ldots, \psi}_{j}, \underbrace{x, \ldots, x}_{k}, \frac{\partial}{\partial x}\rangle,\right. \\
\omega^{\prime} & =\frac{1}{2}(\delta x, \delta x), \alpha^{\prime}=\frac{1}{2}(x, \delta x), \\
\Theta^{\prime} & =\sum_{j \geq 0, k \geq 0, j+k \geq 1} \frac{1}{j !(k+1) !}\left(x, P_{I} \lambda_{j+k}(\underbrace{\psi, \ldots, \psi}_{j}, \underbrace{x, \ldots, x}_{k})\right)
\end{aligned}
$$

where $P_{I}: \mathfrak{h} \rightarrow I$ is the projection to the ideal fixed by the choice of splitting of (37), $\left\{\lambda_{j}\right\}$ are the $L_{\infty}$ operations on $\mathfrak{h}, x$ is the degree $1 I$-valued coordinate on $I[1]$ and (,) is the cyclic pairing on $I$.

(iv) Let $\mathfrak{g}$ be a Lie algebra and suppose there is a Hamiltonian action of $\mathfrak{g}$ on an exact symplectic manifold $\left(M, \omega_{M}=\delta \alpha_{M}\right)$ with equivariant moment map $\mu$ : $M \rightarrow \mathfrak{g}^{*}$. Then we set $\mathcal{M}=\mathfrak{g}[1]$ as in (i), with the structure (32). For the fiber, we set $\mathcal{N}=M$ and

$$
\mathcal{A}=\{\langle\psi, \mu\rangle, \bullet\}, \omega^{\prime}=\omega_{M}, \alpha^{\prime}=\alpha_{M}, \Theta^{\prime}=\langle\psi, \mu\rangle
$$

This is the data of a trivial Hamiltonian $Q$-bundle of degree 0 on $\mathfrak{g}[1] \times M$. Exactness condition for $\omega_{M}$ can be relaxed to the Bohr-Sommerfeld integrality condition, cf. Remark 2 .

(v) For any $Q$-manifold $(\mathcal{M}, Q)$, given a function $\theta \in C^{\infty}(\mathcal{M})$ satisfying

$$
Q \theta=0, \quad|\theta|=p
$$

we can construct a trivial Hamiltonian $Q$-bundle over $\mathcal{M}$ of degree $p-1$ with fiber $\mathcal{N}$ a point, $\mathcal{A}=0, \omega^{\prime}=0, \Theta^{\prime}=\theta$ (and we formally prescribe degree $p-1$ to $\left.\omega^{\prime}\right)$.

\section{BV Pre-observables and Observables}

In this section, we recall some basic structures of BV formalism: classical and quantum BV theory (cf. [18]), observables in classical and quantum setting.

We also introduce the notion of a pre-observable ${ }^{14}$ : an extension of a BV theory by auxiliary fields endowed with an odd-symplectic structure and an action (which is also dependent on the fields of the ambient theory). Given a pre-observable, one can construct an observable by integrating out the auxiliary fields (Propositions 1, 3). Pre-observables come in three versions: purely classical, semi-quantumsuited for integrating out auxiliary fields to produce a classical observable for the

\footnotetext{
${ }^{14}$ This is not a part of the standard BV lore; the term is sometimes used in a totally different sense in the literature.
} 
ambient theory, and quantum — suited for integrating out auxiliary fields to produce a quantum observable for the ambient theory.

Throughout this section in our treatment of quantum objects, we are assuming that the spaces of fields are finite dimensional. This is almost never the case in the interesting examples. In the setting of local quantum field theory, when the spaces of fields are infinite dimensional, measures on fields are problematic to define; however, the BV Laplacians and the integrals over auxiliary fields (which are perturbative path integrals now) can be made sense of with an appropriate regularization/renormalization procedure (one systematic approach is via Wilson's renormalization flow, cf. [11]). Proofs of Propositions 1, 3 cannot be taken for granted in the infinite-dimensional setting, and should be redone within the framework of perturbative path integrals (cf., e.g., the proof of Proposition 6 in Section 6.3).

In view of this, our treatment of quantum objects in this section should be viewed as a simplified discussion, to motivate the interest in pre-observables and argue that given a pre-observable one can expect a path integral expression for an observable.

\subsection{PRE-OBSERVABLES AND OBSERVABLES FOR A CLASSICAL AMBIENT BV THEORY}

DEFINITION 4. We will call a (classical) $B V$ theory a Hamiltonian $Q$-manifold of degree -1 , i.e., a quadruple $(\mathcal{F}, Q, \Omega, S$ ) consisting of a $Q$-manifold $(\mathcal{F}, Q)$ (the space of fields with the BRST operator), the degree -1 symplectic structure (the $B V 2$-form) $\Omega$ and the degree 0 action $S$ satisfying $\{S, \bullet\}_{\Omega}=Q$ and the classical master equation:

$$
\{S, S\}_{\Omega}=0
$$

DEFINITION 5. A (classical) observable for a classical BV theory $(\mathcal{F}, Q, \Omega, S)$ is a degree 0 function $^{15} O \in C^{\infty}(\mathcal{F})$ satisfying

$$
Q(O)=0
$$

Observables $O$ and $O^{\prime}$ are called equivalent if $O^{\prime}-O=Q(\Psi)$ for some $\Psi \in C^{\infty}(\mathcal{F})$, i.e., $O^{\prime}$ and $O$ give the same class in $Q$-cohomology.

DEFINITION 6. For a classical BV theory $(\mathcal{F}, Q, \Omega, S)$ (which we will call the ambient theory), a pre-observable is a degree -1 trivial Hamiltonian $Q$-bundle over $\mathcal{F}$. We will call the fiber the space of auxiliary fields $\mathcal{F}^{\text {aux }}$, which comes with its own degree -1 symplectic structure $\Omega^{\text {aux }}$ and the action for auxiliary fields $S^{\text {aux }} \in$

${ }^{15} \mathrm{We}$ will be assuming degree 0 condition for observables by default, but sometimes it is interesting to relax it. In such cases, we will indicate the degree explicitly. 
$C^{\infty}\left(\mathcal{F} \times \mathcal{F}^{\text {aux }}\right)$ satisfying ${ }^{16}$

$$
Q S^{\text {aux }}+\frac{1}{2}\left\{S^{\text {aux }}, S^{\text {aux }}\right\}_{\Omega^{\text {aux }}}=0
$$

Remark 5. Note that if in addition to (41) we have the property

$$
\left\{S^{\text {aux }}, S^{\text {aux }}\right\}_{\Omega}=0
$$

then the pre-observable gives a new classical BV theory

$$
\left(\mathcal{F} \times \mathcal{F}^{\text {aux }}, Q+\left\{S^{\text {aux }}, \bullet\right\}_{\Omega+\Omega^{\text {aux }}}, \Omega+\Omega^{\text {aux }}, S+S^{\text {aux }}\right)
$$

Note also that the cohomological vector field $Q+\left\{S^{\text {aux }}, \bullet\right\}_{\Omega+\Omega^{\text {aux }}}$ on $\mathcal{F} \times \mathcal{F}^{\text {aux }}$ differs from the one arising from the $Q$-bundle structure by the term $\left\{S^{\text {aux }}, \bullet\right\}_{\Omega}$ and is in general not projectable to $\mathcal{F}$.

In the case when $\mathcal{F}^{\text {aux }}$ is finite dimensional, it makes sense to introduce the following notion.

DEFINITION 7. For a classical BV theory $(\mathcal{F}, Q, \Omega, S)$, a semi-quantum preobservable is a quadruple $\left(\mathcal{F}^{\text {aux }}, \Omega^{\text {aux }}, \mu^{\text {aux }}, S^{\text {aux }}\right)$ consisting of a $\mathbb{Z}$-graded manifold $\mathcal{F}^{\text {aux }}$ and a degree -1 symplectic form $\Omega^{\text {aux }}$ on it. Further,

- $\mathcal{F}^{\text {aux }}$ is endowed with a volume element $\mu^{\text {aux }}$ compatible with $\Omega^{\text {aux }}$ in the sense that the associated BV Laplacian on $C^{\infty}\left(\mathcal{F}^{\text {aux }}\right)$,

$$
\Delta^{\text {aux }}: f \mapsto \frac{1}{2} \operatorname{div}_{\mu^{\text {aux }}}\{f, \bullet\}_{\Omega^{\text {aux }}}
$$

satisfies

$$
\left(\Delta^{\text {aux }}\right)^{2}=0
$$

- Degree 0 action $S^{\text {aux }} \in C^{\infty}\left(\mathcal{F} \times \mathcal{F}^{\text {aux }}\right)$ satisfies

$$
Q S^{\text {aux }}+\frac{1}{2}\left\{S^{\text {aux }}, S^{\text {aux }}\right\}_{\Omega^{\text {aux }}}-i \Delta^{\text {aux }} S^{\text {aux }}=0
$$

or, equivalently,

$$
\left(Q-i \Delta^{\text {aux }}\right) \mathrm{e}^{i S^{\text {aux }}}=0
$$

${ }^{16}$ In the notations of Definition 2, here $\mathcal{N}=\mathcal{F}^{\text {aux }}, \omega^{\prime}=\Omega^{\text {aux }}, \Theta^{\prime}=S^{\text {aux }} ; Q$ is understood as the horizontal lift of the cohomological vector field on $\mathcal{F}$ to the total space $\mathcal{F} \times \mathcal{F}$ aux. The total cohomological vector field on $\mathcal{F} \times \mathcal{F}^{\text {aux }}$, as defined by the $Q$-bundle structure, is $Q^{\text {tot }}=Q+\left\{S^{\text {aux }}, \bullet\right\}_{\Omega^{\text {aux }}}$. 
We call two semi-quantum pre-observables $\left(\mathcal{F}^{\text {aux }}, \Omega^{\text {aux }}, \mu^{\text {aux }}, S^{\text {aux }}\right)$ and $\left(\mathcal{F}^{\text {aux }}, \Omega^{\text {aux }}, \mu^{\text {aux }}, \tilde{S}^{\text {aux }}\right)$ equivalent, if there exists a degree -1 function $R^{\text {aux }} \in$ $C^{\infty}\left(\mathcal{F} \times \mathcal{F}^{\text {aux }}\right)$ such that

$$
\mathrm{e}^{i \tilde{S}^{\text {aux }}}-\mathrm{e}^{i S^{\text {aux }}}=\left(Q-i \Delta^{\text {aux }}\right)\left(\mathrm{e}^{i S^{\text {aux }}} R^{\text {aux }}\right)
$$

Infinitesimally, this equivalence condition can be written as

$$
\tilde{S}^{\text {aux }}-S^{\text {aux }}=Q R^{\text {aux }}+\left\{S^{\text {aux }}, R^{\text {aux }}\right\}_{\Omega^{\text {aux }}}-i \Delta^{\text {aux }} R^{\text {aux }}+\mathcal{O}\left(R^{2}\right)
$$

In particular, a classical pre-observable can be promoted to a semi-quantum preobservable if there exists a volume element $\mu^{\text {aux }}$ on $\mathcal{F}^{\text {aux }}$ compatible with $\Omega^{\text {aux }}$, such that in addition to (41) we have $\Delta^{\text {aux }} S^{\text {aux }}=0$.

Given a semi-quantum pre-observable, one can construct an observable for the ambient classical BV theory using fiber BV integrals [14] as follows.

PROPOSITION 1. Given a semi-quantum pre-observable ( $\left.\mathcal{F}^{\text {aux }}, \Omega^{\text {aux }}, \mu^{\text {aux }}, S^{\text {aux }}\right)$, set

$$
O_{\mathcal{L}}=\left.\int_{\mathcal{L} \subset \mathcal{F} \text { aux }} \mathrm{e}^{i S^{\text {aux }}} \sqrt{\mu^{\text {aux }}}\right|_{\mathcal{L}} \in C^{\infty}(\mathcal{F})
$$

for $\mathcal{L} \subset \mathcal{F}^{\text {aux }}$ a Lagrangian submanifold. Then:

(i) $O_{\mathcal{L}}$ is an observable, i.e., $Q\left(O_{\mathcal{L}}\right)=0$.

(ii) If Lagrangian submanifolds $\mathcal{L}$ and $\mathcal{L}^{\prime}$ can be connected by a Lagrangian homotopy, then the difference $O_{\mathcal{L}^{\prime}}-O_{\mathcal{L}}$ is $Q$-exact, i.e., observables $O_{\mathcal{L}}$ and $O_{\mathcal{L}^{\prime}}$ are equivalent.

(iii) Given two equivalent semi-quantum pre-observables $S^{\text {aux }}$ and $\tilde{S}^{\text {aux }}$, the associated observables $O_{\mathcal{L}}$ and $\tilde{O}_{\mathcal{L}}$ are equivalent.

Proof. Applying $Q$ to the integral (46), we obtain

$$
Q\left(O_{\mathcal{L}}\right)=\left.Q \int_{\mathcal{L}} \mathrm{e}^{i S^{\text {aux }}} \sqrt{\mu^{\text {aux }}}\right|_{\mathcal{L}}=\left.\int_{\mathcal{L}}\left(Q-i \Delta^{\text {aux }}\right) \mathrm{e}^{i S^{\text {aux }}} \sqrt{\mu^{\text {aux }}}\right|_{\mathcal{L}}=0
$$

where we used (43) and the Stokes' theorem for BV integrals [18], that the BV integral of a $\Delta$-coboundary vanishes. ${ }^{17}$ This proves (i).

17 Recall that the version of Stokes' theorem for BV integrals (theorems 1 and 2 in [18]) asserts that for an odd-symplectic supermanifold $\mathcal{M}$ with compact body endowed with a compatible volume element $\mu$, one has the following. (i) For $f \in C^{\infty}(\mathcal{M})$ and $\mathcal{L} \subset \mathcal{M}$ a Lagrangian submanifold, $\left.\int_{\mathcal{L}} \Delta(f) \cdot \sqrt{\mu}\right|_{\mathcal{L}}=0$. (ii) If $\mathcal{L}$ and $\tilde{\mathcal{L}}$ are two Lagrangians in $\mathcal{M}$ with homologous bodies and $f \in C^{\infty}(\mathcal{M})$ satisfies $\Delta f=0$, then $\left.\int_{\mathcal{L}} f \sqrt{\mu}\right|_{\mathcal{L}}=\left.\int_{\tilde{\mathcal{L}}} f \sqrt{\mu}\right|_{\mathcal{L}}$. Note that the assumption of (ii) holds in particular if there exists a smooth family of Lagrangians connecting $\mathcal{L}$ and $\tilde{\mathcal{L}}$. In the setting of [18], Lagrangians are assumed to be closed. The usual proof goes via reduction, by the odd Fourier transform, to the ordinary Stokes' theorem for integration of forms over chains. The proof of the statement above goes through in greater generality: assumption of $\mathcal{M}$ being compact and Lagrangians being closed can be dropped, as long as the integrals converge and asymptotic boundary terms vanish. 
Now let $\left\{\mathcal{L}_{t}\right\}_{t \in[0,1]}$ be a smooth family of Lagrangian submanifolds of $\left(\mathcal{F}^{\text {aux }}, \Omega^{\text {aux }}\right.$ ) connecting $\mathcal{L}=\mathcal{L}_{0}$ and $\mathcal{L}^{\prime}=\mathcal{L}_{1}$. An infinitesimal deformation of a Lagrangian submanifold $\mathcal{L}_{t}$ is given by a graph of a Hamiltonian vector field ${ }^{18}$

$$
\text { “ } \frac{\mathrm{d}}{\mathrm{d} t} \mathcal{L}_{t} "=\operatorname{graph}\left(\left\{\bullet, \Psi_{t}\right\}_{\Omega^{\mathrm{aux}}}\right) \in \Gamma\left(\mathcal{L}_{t}, N \mathcal{L}_{t}\right)
$$

viewed as a section of the normal bundle of $\mathcal{L}_{t}$, with $\Psi_{t} \in C^{\infty}\left(\mathcal{L}_{t}\right),\left|\Psi_{t}\right|=-1$ the generator. Hence,

$$
\begin{aligned}
\frac{\mathrm{d}}{\mathrm{d} t} O_{\mathcal{L}_{t}} & =\frac{\mathrm{d}}{\mathrm{d} t} \int_{\mathcal{L}_{t}} \mathrm{e}^{i S^{\text {aux }}\left|\mathcal{L}_{t} \sqrt{\mu^{\text {aux }}}\right| \mathcal{L}_{t}} \\
& =\int_{\mathcal{L}_{t}}\left(\left\{\mathrm{e}^{i S^{\text {aux }}}, \Psi_{t}\right\}_{\Omega^{\text {aux }}}+\mathrm{e}^{i S^{\text {aux }}} \cdot \Delta^{\text {aux }}\left(\Psi_{t}\right)\right) \cdot \sqrt{\mu^{\text {aux }}} \mathcal{L}_{t} \\
& =\int_{\mathcal{L}_{t}}\left(\Delta^{\text {aux }}\left(\mathrm{e}^{i S^{\text {aux }}} \Psi_{t}\right)-\Delta^{\text {aux }}\left(\mathrm{e}^{i S^{\text {aux }}}\right) \cdot \Psi_{t}\right) \cdot \sqrt{\mu^{\text {aux }}} \mid \mathcal{L}_{t} \\
& =\int_{\mathcal{L}_{t}}\left(\Delta^{\text {aux }}\left(\mathrm{e}^{i S^{\text {aux }}} \Psi_{t}\right)+i Q\left(\mathrm{e}^{i S^{\text {aux }}}\right) \cdot \Psi_{t}\right) \cdot \sqrt{\mu^{\text {aux }}} \mathcal{L}_{t} \\
& =Q\left(i \int_{\mathcal{L}_{t}} \mathrm{e}^{i S^{\text {aux }}} \Psi_{t} \cdot \sqrt{\mu^{\text {aux }}} \mid \mathcal{L}_{t}\right)
\end{aligned}
$$

The term $\Delta^{\text {aux }}\left(\Psi_{t}\right)$ in the second line comes from the transformation of measure $\left.\sqrt{\mu^{\text {aux }}}\right|_{\mathcal{L}_{t}}$ by the infinitesimal shift of Lagrangian (48). This calculation implies that

$$
O_{\mathcal{L}^{\prime}}-O_{\mathcal{L}}=Q\left(\int_{0}^{1} \mathrm{~d} t i \int_{\mathcal{L}_{t}} \mathrm{e}^{i S^{\text {aux }}} \Psi_{t} \cdot \sqrt{\mu^{\text {aux }}} \mathcal{L}_{t}\right)
$$

and thus proves (ii).

Item (iii) follows immediately from the construction (46), our definition of equivalence (44) and the Stokes' theorem for BV integrals:

$$
\begin{aligned}
\tilde{O}_{\mathcal{L}}-O_{\mathcal{L}} & =\int_{\mathcal{L}}\left(\mathrm{e}^{i \tilde{S}^{\text {aux }}}-\mathrm{e}^{i S^{\text {aux }}}\right) \sqrt{\mu^{\text {aux }}} \mathcal{L}_{\mathcal{L}} \\
& =\left.\int_{\mathcal{L}}\left(Q-i \Delta^{\text {aux }}\right)\left(\mathrm{e}^{i S^{\text {aux }}} R^{\text {aux }}\right) \sqrt{\mu^{\text {aux }}}\right|_{\mathcal{L}}=Q\left(\left.\int_{\mathcal{L}} \mathrm{e}^{i S^{\text {aux }}} R^{\text {aux }} \sqrt{\mu^{\text {aux }}}\right|_{\mathcal{L}}\right)
\end{aligned}
$$

Here, we are implicitly assuming convergence of the integral (46), and for the proof of (ii) the integral over $\mathcal{L}_{t}$ should converge for every $t \in[0,1]$, cf. footnote 17.

The following construction allows one to produce new semi-quantum preobservables out of old ones by partially integrating out the auxiliary fields.

${ }^{18}$ A general infinitesimal Lagrangian deformation is given by $\operatorname{graph}\left(l_{\left(\Omega^{\text {aux }}\right)^{-1}} \chi_{t}\right)$ for any degree -1 closed 1 -form $\chi_{t} \in \Omega^{1}\left(\mathcal{L}_{t}\right)$. Since in non-zero degree a closed form is automatically exact (cf. Remark 1), we have (48) with the generator $\Psi_{t}=\iota_{E} \chi_{t}$. 
PROPOSITION 2. Given a semi-quantum pre-observable $\left(\mathcal{F}^{\text {aux }}, \Omega^{\text {aux }}, \mu^{\text {aux }}, S^{\text {aux }}\right)$, assume that $\mathcal{F}^{\text {aux }}$ is given as a product, ${ }^{19} \mathcal{F}^{\text {aux }}=\mathcal{F}_{\mathrm{Z}}^{\text {aux }} \times \tilde{\mathcal{F}}^{\text {aux }}$, so that $\Omega^{\text {aux }}$ and $\mu^{\text {aux }}$ split:

$$
\Omega^{\mathrm{aux}}=\Omega_{\mathrm{z}}^{\mathrm{aux}}+\tilde{\Omega}^{\mathrm{aux}}, \quad \mu^{\mathrm{aux}}=\mu_{\mathrm{z}}^{\mathrm{aux}} \times \tilde{\mu}^{\text {aux }}
$$

Define $S_{\mathrm{z}}^{\text {aux }} \in C^{\infty}\left(\mathcal{F} \times \mathcal{F}_{\mathrm{z}}^{\text {aux }}\right)$ by

$$
\mathrm{e}^{i S_{\mathrm{Z}}^{\text {aux }}}=\left.\int_{\tilde{\mathcal{L}} \subset \tilde{\mathcal{F}} \text { aux }} \mathrm{e}^{i S^{\text {aux }}} \sqrt{\tilde{\mu}^{\text {aux }}}\right|_{\mathcal{L}}
$$

with $\tilde{\mathcal{L}}$ a Lagrangian submanifold of $\left(\tilde{\mathcal{F}}^{\text {aux }}, \tilde{\Omega}^{\text {aux }}\right)$. Then

(i) $\left(\mathcal{F}_{\mathrm{Z}}^{\mathrm{aux}}, \Omega_{\mathrm{z}}^{\mathrm{aux}}, \mu_{\mathrm{Z}}^{\text {aux }}, S_{\mathrm{Z}}^{\mathrm{aux}}\right)$ is a semi-quantum pre-observable for the same ambient classical $B V$ theory.

(ii) The observable for the ambient theory induced from $S_{z}^{\text {aux }}$ using (46) with Lagrangian $\mathcal{L}_{\mathrm{Z}} \subset \mathcal{F}_{\mathrm{Z}}^{\mathrm{aux}}$ is equivalent to the one induced directly from $S^{\text {aux }}$ using Lagrangian $\mathcal{L} \subset \mathcal{F}^{\text {aux }}$, provided that $\mathcal{L}$ can be connected with $\mathcal{L}_{\mathrm{z}} \times \tilde{\mathcal{L}}$ by a Lagrangian homotopy in $\mathcal{F}^{\text {aux }}$.

Proof. Similar to (47), we have

$$
\left(Q-i \Delta_{\mathrm{z}}^{\mathrm{aux}}\right) \mathrm{e}^{i S_{\mathrm{z}}^{\text {aux }}}=\left.\int_{\tilde{\mathcal{L}}}(\underbrace{-i \Delta_{\mathrm{z}}^{\mathrm{aux}}-i \tilde{\Delta}^{\mathrm{aux}}}_{-i \Delta^{\text {aux }}}) \mathrm{e}^{i \text { aux }} \sqrt{\mu^{\text {aux }}}\right|_{\tilde{\mathcal{L}}}=0
$$

which proves (i). Item (ii) is an immediate corollary of (ii) of Proposition 1.

\subsection{PRE-OBSERVABLES AND OBSERVABLES FOR A QUANTUM AMBIENT BV THEORY}

In the case when the space of fields of the ambient theory $\mathcal{F}$ is finite dimensional, it makes sense to introduce the following notions.

DEFINITION 8. (cf. [18]) A quantum $B V$ theory is a quadruple $(\mathcal{F}, \Omega, \mu, S)$ where $(\mathcal{F}, \Omega)$ is a $\mathbb{Z}$-graded manifold with a degree -1 symplectic form $\Omega ; \mu$ is a volume element on $\mathcal{F}$, compatible with $\Omega$ as in Definition 7, i.e., the BV Laplacian $\Delta: f \mapsto \frac{1}{2} \operatorname{div}_{\mu}\{f, \bullet\}_{\Omega}$ satisfies $\Delta^{2}=0$; the degree 0 action $S \in C^{\infty}(\mathcal{F})$ is required to satisfy the quantum master equation

$$
\frac{1}{2}\{S, S\}_{\Omega}-i \Delta S=0
$$

or, equivalently,

$$
\Delta \mathrm{e}^{i S}=0
$$

\footnotetext{
${ }^{19}$ Subscript "z" refers to auxiliary zero modes.
} 
For a quantum BV theory, it is convenient to introduce a degree 1 second-order differential operator

$$
\delta_{\mathrm{BV}}=\{S, \bullet\}_{\Omega}-i \Delta=\mathrm{e}^{-i S}(-i \Delta) \mathrm{e}^{i S}
$$

which satisfies $\left(\delta_{\mathrm{BV}}\right)^{2}=0$.

DEFINITION 9. A quantum observable ${ }^{20}$ for a quantum BV theory is a degree 0 function $O \in C^{\infty}(\mathcal{F})$ satisfying

$$
\delta_{\mathrm{BV}} O=0
$$

which is equivalent to

$$
\Delta\left(O \mathrm{e}^{i S}\right)=0
$$

Observables $O$ and $O^{\prime}$ are called equivalent if $O^{\prime}-O=\delta_{\mathrm{BV}}(\Psi)$ for some $\Psi \in$ $C^{\infty}(\mathcal{F})$, i.e. $O^{\prime}$ and $O$ give the same class in $\delta_{\mathrm{BV}}$-cohomology.

Given a quantum observable, one can define its correlator as

$$
\langle O\rangle_{\mathcal{L}}=\frac{\left.\int_{\mathcal{L} \subset \mathcal{F}} O \mathrm{e}^{i S} \sqrt{\mu}\right|_{\mathcal{L}}}{\left.\int_{\mathcal{L} \subset \mathcal{F}} \mathrm{e}^{i S} \sqrt{\mu}\right|_{\mathcal{L}}} \in \mathbb{C}
$$

where $\mathcal{L}$ is a Lagrangian submanifold of $(\mathcal{F}, \Omega)$ and we are assuming convergence. By the Stokes' theorem for BV integrals, this expression does not change with Lagrangian homotopy of $\mathcal{L}:\langle O\rangle_{\mathcal{L}}=\langle O\rangle_{\mathcal{L}^{\prime}}$, and also correlators of equivalent observables coincide: $\langle O\rangle_{\mathcal{L}}=\left\langle O^{\prime}\right\rangle_{\mathcal{L}}$, cf. [18].

Finally, if both $\mathcal{F}$ and $\mathcal{F}^{\text {aux }}$ are finite dimensional, the following definition makes sense.

DEFINITION 10. A quantum pre-observable for a quantum BV theory is a semiquantum pre-observable $\left(\mathcal{F}^{\text {aux }}, \Omega^{\text {aux }}, \mu^{\text {aux }}, S^{\text {aux }}\right)$ where instead of Eq. (42) we require that $S+S^{\text {aux }}$ satisfies the quantum master equation on $\mathcal{F} \times \mathcal{F}^{\text {aux }}$ :

$$
\left(\Delta+\Delta^{\text {aux }}\right) \mathrm{e}^{i\left(S+S^{\text {aux }}\right)}=0
$$

The analog of Proposition 1 in the setting of quantum BV theory is as follows.

\footnotetext{
${ }^{20}$ Here, we define a quantum observable in the framework of Lagrangian field theory in BV formalism, as something that can be averaged over the space of fields to yield a correlator (50). In other contexts one has quite different definitions of quantum observables: e.g., in the framework of Atiyah's topological quantum field theory [13], an observable is a pair consisting of a submanifold $\gamma \subset \Sigma$ of the spacetime manifold and a vector $\hat{O}_{\gamma}$ in the space of states associated to the boundary of a tubular neighborhood of $\gamma$ in $\Sigma$. The passage from the first picture to the second one goes through path integral quantization of the ambient topological theory on the tubular neighborhood (as on a manifold with boundary) with the insertion of observable.
} 
PROPOSITION 3. Given a quantum pre-observable ( $\left.\mathcal{F}^{\text {aux }}, \Omega^{\text {aux }}, \mu^{\text {aux }}, S^{\text {aux }}\right)$, define again $O_{\mathcal{L}}$ as

$$
O_{\mathcal{L}}=\left.\int_{\mathcal{L} \subset \mathcal{F} \text { aux }} \mathrm{e}^{i S^{\text {aux }}} \sqrt{\mu^{\text {aux }}}\right|_{\mathcal{L}} \in C^{\infty}(\mathcal{F})
$$

for $\mathcal{L} \subset \mathcal{F}^{\text {aux }}$ a Lagrangian submanifold. Then:

(i) $O_{\mathcal{L}}$ is a quantum observable, i.e., $\delta_{\mathrm{BV}} O_{\mathcal{L}}=0$.

(ii) If Lagrangian submanifolds $\mathcal{L}$ and $\mathcal{L}^{\prime}$ can be connected by a Lagrangian homotopy, then the difference $O_{\mathcal{L}^{\prime}}-O_{\mathcal{L}}$ is $\delta_{\mathrm{BV}}$ exact.

Proof. The proof is obtained from the proof of Proposition 1 by replacing $Q$ by $-i \Delta, S^{\text {aux }}$ by $S+S^{\text {aux }}$ and $O$ by $O \mathrm{e}^{i S}$ everywhere.

Similarly, Proposition 2 holds in the context of quantum pre-observables.

Remark 6. It is customary for perturbative quantization to introduce a formal parameter (the "Planck's constant") $\hbar$ by making a rescaling

$$
\Omega \rightarrow \hbar^{-1} \Omega, \quad S \rightarrow \hbar^{-1} S
$$

(which means $\Omega^{\text {old }}=\hbar^{-1} \Omega^{\text {new }}$, etc.) where the new action is a formal power series in $\hbar, S \in C^{\infty}(\mathcal{F})[[\hbar]]$. Hamiltonian vector field $\{S, \bullet\}_{\Omega}$ does not rescale and the BV Laplacian rescales as $\Delta \rightarrow \hbar \Delta$. For the structure on auxiliary fields, one does the same:

$$
\Omega^{\text {aux }} \rightarrow \hbar^{-1} \Omega^{\text {aux }}, \quad S^{\text {aux }} \rightarrow \hbar^{-1} S^{\text {aux }}
$$

With these redefinitions the Eq. (42) on semi-quantum pre-observables becomes

$$
Q S^{\text {aux }}+\frac{1}{2}\left\{S^{\text {aux }}, S^{\text {aux }}\right\}_{\Omega^{\text {aux }}}-i \hbar \Delta^{\text {aux }} S^{\text {aux }}=0
$$

and can be solved by obstruction theory order by order in $\hbar$ for $S^{\text {aux }}=S_{(0)}^{\text {aux }}+$ $\hbar S_{(1)}^{\text {aux }}+\hbar^{2} S_{(2)}^{\text {aux }}+\cdots$ starting from $S_{(0)}^{\text {aux }}$ a solution of (41). Likewise, the BV pushforward formula (46) becomes

$$
O=\left.\int_{\mathcal{L} \subset \mathcal{F} \text { aux }} \mathrm{e}^{\frac{i}{\hbar} S^{\text {aux }}} \sqrt{\mu^{\text {aux }}}\right|_{\mathcal{L}}
$$

and can be evaluated by the stationary phase formula.

Note that one can also introduce two independent Planck's constants $\hbar, \hbar^{\text {aux }}$ for the ambient (51) and auxiliary (52) theory respectively. 


\section{AKSZ Pre-observable Associated to a Hamiltonian $Q$-Bundle}

Let $(\mathcal{M}, Q, \omega=\delta \alpha, \Theta)$ be a Hamiltonian $Q$-manifold and $\Sigma$ a closed oriented spacetime manifold, $\operatorname{dim} \Sigma=|\omega|+1$. Then, we have the AKSZ theory with

$$
\begin{aligned}
& \mathcal{F}_{\Sigma}=\operatorname{Map}(T[1] \Sigma, \mathcal{M}), \quad Q_{\Sigma}=\left(d_{\Sigma}\right)^{\text {lifted }}+Q^{\text {lifted }}, \\
& \Omega_{\Sigma}=(-1)^{\operatorname{dim} \Sigma} \tau_{\Sigma}(\omega), \quad S_{\Sigma}=\iota_{\Sigma} d_{\Sigma}^{\text {lifted }} \tau_{\Sigma}(\alpha)+\tau_{\Sigma}(\Theta)
\end{aligned}
$$

as in Section 2.

Given a trivial Hamiltonian $Q$-bundle over $\mathcal{M}$ with fiber data $\left(\mathcal{N}, \mathcal{A}, \omega^{\prime}=\right.$ $\delta \alpha^{\prime}, \Theta^{\prime}$ ), a closed oriented manifold $\gamma$ of dimension $\operatorname{dim} \gamma=\left|\omega^{\prime}\right|+1$ and a smooth $\operatorname{map}^{21} \mathrm{i}: \gamma \rightarrow \Sigma$, we can construct a pre-observable for the AKSZ theory (53) with target $\mathcal{M}$ as follows. Set

$$
\begin{aligned}
& \mathcal{F}_{\gamma}=\operatorname{Map}(T[1] \gamma, \mathcal{N}), \quad \mathcal{A}_{\gamma}=\left(d_{\gamma}\right)^{\text {lifted }}+p^{*} \mathcal{A}^{\text {lifted }}, \\
& \Omega_{\gamma}=(-1)^{\operatorname{dim} \gamma} \tau_{\gamma}\left(\omega^{\prime}\right), \quad S_{\gamma}=\iota_{\left(d_{\gamma}\right)} \text { lifted } \tau_{\gamma}\left(\alpha^{\prime}\right)+p^{*} \tau_{\gamma}^{\text {tot }}\left(\Theta^{\prime}\right)
\end{aligned}
$$

Here, the notations are as follows.

- $\tau_{\gamma}: \Omega^{\bullet}(\mathcal{N}) \rightarrow \Omega^{\bullet}\left(\mathcal{F}_{\gamma}\right)$ and $\tau_{\gamma}^{\text {tot }}: \Omega^{\bullet}(\mathcal{E}) \rightarrow \Omega^{\bullet}(\operatorname{Map}(T[1] \gamma, \mathcal{E}))$ are the transgression maps, defined as in (11); $\mathcal{E}=\mathcal{M} \times \mathcal{N}$ is the total space of the target Hamiltonian $Q$-bundle.

- Map $p=\mathrm{i}^{*}: \mathcal{F}_{\Sigma} \rightarrow \operatorname{Map}(T[1] \gamma, \mathcal{M})$ is the pull-back of ambient fields by $\mathrm{i}: \gamma \rightarrow \Sigma$ and $p^{*}: C^{\infty}(\operatorname{Map}(T[1] \gamma, \mathcal{M})) \rightarrow C^{\infty}\left(\mathcal{F}_{\Sigma}\right)$ is the pull-back by $p$. By the trivial extension to auxiliary fields, $p=p \times \operatorname{id}_{\mathcal{F}_{\gamma}}$ also maps $\mathcal{F}_{\Sigma} \times \mathcal{F}_{\gamma}$ to $\operatorname{Map}(T[1] \gamma, \mathcal{E})$.

- $\left(d_{\gamma}\right)^{\text {lifted }} \in \mathfrak{X}\left(\mathcal{F}_{\gamma}\right) \subset \mathfrak{X}^{\text {vert }}\left(\mathcal{F}_{\Sigma} \times \mathcal{F}_{\gamma}\right)$ is the lifting of the de Rham differential on $\gamma$ to a vector field on the mapping space $\operatorname{Map}(T[1] \gamma, \mathcal{N})$.

- $\mathcal{A}^{\text {lifted }}$ is the lifting of the vertical vector field $\mathcal{A} \in \mathfrak{X}^{\text {vert }}(\mathcal{E})$ to a vertical vector field on the mapping space $\operatorname{Map}(T[1] \gamma, \mathcal{E})$, so that $p^{*} \mathcal{A}^{\text {lifted }}$ becomes a vertical vector field on $\mathcal{F}_{\Sigma} \times \mathcal{F}_{\gamma}$.

PROPOSITION 4. The data (54) define a classical pre-observable for the AKSZ theory (53), in particular

$$
Q_{\Sigma} S_{\gamma}+\frac{1}{2}\left\{S_{\gamma}, S_{\gamma}\right\}_{\Omega_{\gamma}}=0
$$

Proof. First, let us check that $\mathcal{A}_{\gamma}$ as defined in (54) is the Hamiltonian vector field for $S_{\gamma}$. Introduce the notations for the source (kinetic) and target parts of $\mathcal{A}_{\gamma}$ and $S_{\gamma}$ :

$$
\mathcal{A}_{\gamma}=\underbrace{\left(d_{\gamma}\right)^{\text {lifted }}}_{\mathcal{A}_{\gamma}^{\text {kin }}}+\underbrace{p^{*} \mathcal{A}^{\text {lifted }}}_{\mathcal{A}_{\gamma}^{\text {target }}}, \quad S_{\gamma}=\underbrace{\iota\left(d_{\gamma}\right)^{\text {lifted }} \tau_{\gamma}\left(\alpha^{\prime}\right)}_{S_{\gamma}^{\text {kin }}}+\underbrace{p^{*} \tau_{\gamma}^{\text {tot }}\left(\Theta^{\prime}\right)}_{S_{\gamma}^{\text {target }}}
$$

\footnotetext{
${ }^{21} \mathrm{By}$ default, we assume that $\mathrm{i}$ is an embedding, cf. Remark 10 below.
} 
Then, we have

$$
\begin{aligned}
& \iota_{\mathcal{A}_{\gamma}^{\text {kin }}} \Omega_{\gamma}=\iota_{\left(d_{\gamma}\right)} \operatorname{lifted}(-1)^{\operatorname{dim} \gamma} \tau_{\gamma}\left(\omega^{\prime}\right)=\iota_{\left(d_{\gamma}\right)}{ }^{\operatorname{lifted}} \delta \tau_{\gamma}\left(\alpha^{\prime}\right)
\end{aligned}
$$

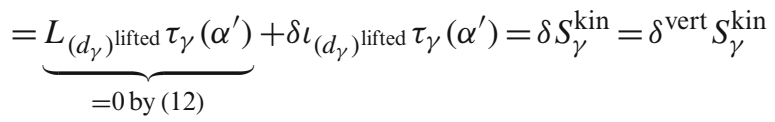

and

$$
\begin{aligned}
{ }^{\iota_{\mathcal{A}}} \mathcal{A}_{\gamma}^{\text {target }} \Omega_{\gamma} & =p^{*} \iota_{\mathcal{A}^{\text {lifted }}}(-1)^{\operatorname{dim} \gamma} \tau_{\gamma}^{\text {tot }}\left(\omega^{\prime}\right) \\
& =(-1)^{\operatorname{dim} \gamma} p^{*} \tau_{\gamma}^{\text {tot }}(\underbrace{\iota_{\mathcal{A}} \omega^{\prime}}_{=\delta^{\text {vert }} \Theta^{\prime}})=\delta^{\text {vert }} p^{*} \tau_{\gamma}^{\text {tot }}\left(\Theta^{\prime}\right)=\delta^{\text {vert }} S_{\gamma}^{\text {target }}
\end{aligned}
$$

Here, $\delta^{\text {vert }}$ stands for the de Rham differential in the fiber direction, as in Section 3.3.

Collecting $(56,57)$, we get

$$
\left\{S_{\gamma}, \bullet\right\}_{\Omega_{\gamma}}=\mathcal{A}_{\gamma}
$$

Next, let us prove (55). Note that

$$
Q_{\Sigma} S_{\gamma}^{\mathrm{kin}}=0
$$

since $S_{\gamma}^{\text {kin }}$ does not depend on the ambient fields (i.e., is a function on $\mathcal{F}_{\Sigma} \times \mathcal{F}_{\gamma}$, constant in the direction of $\mathcal{F}_{\Sigma}$ ); also

$$
Q_{\Sigma}^{\mathrm{kin}} S_{\gamma}^{\mathrm{target}}=p^{*}\left(\left.Q_{\Sigma}^{\mathrm{kin}}\right|_{\gamma} \tau_{\gamma}^{\mathrm{tot}}\left(\Theta^{\prime}\right)\right)=0
$$

as in (12), where $\left.Q_{\Sigma}^{\mathrm{kin}}\right|_{\gamma}$ is the lifting of $d_{\gamma}$ to $\operatorname{Map}(T[1] \gamma, \mathcal{M})$, extended to a horizontal vector field on $\operatorname{Map}(T[1] \gamma, \mathcal{E})$.

Thus

$$
\begin{aligned}
& Q_{\Sigma} S_{\gamma}+\frac{1}{2}\left\{S_{\gamma}, S_{\gamma}\right\}_{\Omega_{\gamma}} \\
& =Q_{\Sigma}^{\text {target }} S_{\gamma}^{\text {target }}+\frac{1}{2}\left\{S_{\gamma}^{\text {kin }}, S_{\gamma}^{\text {kin }}\right\}_{\Omega_{\gamma}}+\left\{S_{\gamma}^{\text {kin }}, S_{\gamma}^{\text {target }}\right\}+\frac{1}{2}\left\{S_{\gamma}^{\text {target }}, S_{\gamma}^{\text {target }}\right\}_{\Omega_{\gamma}} \\
& =\frac{1}{2}\left\{S_{\gamma}^{\text {kin }}, S_{\gamma}^{\text {kin }}\right\}_{\Omega_{\gamma}}+\underbrace{\mathcal{A}_{\gamma}^{\text {kin }} S_{\gamma}^{\text {target }}}_{0 \text { by }(12)}+\left(Q_{\Sigma}^{\text {target }}+\frac{1}{2} \mathcal{A}_{\gamma}^{\text {target }}\right) S_{\gamma}^{\text {target }}
\end{aligned}
$$

Here, the first term on the r.h.s. has $\left(\left(d_{\gamma}\right)^{\text {lifted }}\right)^{2}=0$ as its Hamiltonian vector field on $\mathcal{F}_{\gamma}$, thus it is a degree 1 constant function on $\mathcal{F}_{\gamma}$, and hence vanishes. Therefore, continuing the calculation (58) and using (13), we have

$$
\begin{aligned}
Q_{\Sigma} S_{\gamma}+\frac{1}{2}\left\{S_{\gamma}, S_{\gamma}\right\}_{\Omega_{\gamma}} & =(-1)^{\operatorname{dim} \gamma} p^{*} \tau_{\gamma}^{\text {tot }}\left(Q \Theta^{\prime}+\frac{1}{2} \mathcal{A} \Theta^{\prime}\right) \\
& =(-1)^{\operatorname{dim} \gamma} p^{*} \tau_{\gamma}^{\text {tot }}\left(Q \Theta^{\prime}+\frac{1}{2}\left\{\Theta^{\prime}, \Theta^{\prime}\right\}_{\omega^{\prime}}\right)=0
\end{aligned}
$$


since the target is a Hamiltonian $Q$-bundle and Eq. (30) holds there. This finishes the proof.

In coordinates. Alongside with the superfield (18) for the ambient theory on $\Sigma$, we now have a superfield for the auxiliary theory on $\gamma$ :

$$
\begin{aligned}
& Y^{i}\left(v^{1}, \ldots, v^{\operatorname{dim} \gamma}, \xi^{1}, \ldots, \xi^{\operatorname{dim} \gamma}\right) \\
& =\sum_{k=1}^{\operatorname{dim} \gamma} \underbrace{\sum_{1 \leq \nu_{1}<\cdots<v_{k} \leq \operatorname{dim} \gamma} Y_{\nu_{1} \cdots v_{k}}^{i}\left(v^{1}, \ldots, v^{\operatorname{dim} \gamma}\right) \xi^{\nu_{1}} \ldots \xi^{\nu_{k}}}_{Y_{(k)}^{i}(v, \xi)}
\end{aligned}
$$

associated to local homogeneous coordinates $y^{i}$ on $\mathcal{N}$.

Here, $v^{1}, \ldots, v^{\operatorname{dim} \gamma}$ are local coordinates on $\gamma$ and $\xi^{v}$ stands for $d v^{v}, v=$ $1, \ldots, \operatorname{dim} \gamma$.

Let $\alpha^{\prime}$ and $\omega^{\prime}$ be locally given on $\mathcal{N}$ as $\alpha^{\prime}=\alpha_{i}^{\prime}(y) \delta y^{i}, \omega^{\prime}=\frac{1}{2} \omega_{i j}^{\prime}(y) \delta y^{i} \wedge \delta y^{j}$. Then the BV 2-form for auxiliary fields is:

$$
\Omega_{\gamma}=(-1)^{\operatorname{dim} \gamma} \int_{\gamma} \frac{1}{2} \omega_{i j}^{\prime}(Y) \delta Y^{i} \wedge \delta Y^{j}
$$

Its primitive is:

$$
\alpha_{\gamma}=\int_{\gamma} \alpha_{i}^{\prime}(Y) \delta Y^{i}
$$

The action for auxiliary fields is:

$$
S_{\gamma}=\int_{\gamma} \alpha_{i}^{\prime}(Y) d Y^{i}+\int_{\gamma} \Theta^{\prime}\left(\mathrm{i}^{*} X, Y\right)
$$

Vertical part of the cohomological vector field $\mathcal{A}_{\gamma}$ acts on the auxiliary superfield by

$$
\mathcal{A}_{\gamma} Y^{i}=d Y^{i}+\mathcal{A}^{i}\left(i^{*} X, Y\right)
$$

if on the target we have $\mathcal{A}=\mathcal{A}^{i}(x, y) \frac{\partial}{\partial y^{i}}$.

Remark 7. In the case when $i(\gamma) \subset \Sigma$ is an embedded submanifold of positive codimension, the expression

$$
\left\{S_{\gamma}, S_{\gamma}\right\}_{\Omega_{\Sigma}}
$$

is ill-defined, because it formally contains $\int_{\Sigma}\left(\delta_{\gamma}\right)^{2} \cdots$ where $\delta_{\gamma}$ is the delta function supported on $i(\gamma) \subset \Sigma$. On the other hand, if we use adapted local coordinates $u^{1}, \ldots, u^{\operatorname{dim} \Sigma}$ on $\Sigma$ in which $i(\gamma)$ is given by $u^{\operatorname{dim} \gamma+1}=\cdots=u^{\operatorname{dim} \Sigma}=0$, the auxiliary action $S_{\gamma}$ only depends on the components of the ambient superfield 
$X_{\mu_{1} \cdots \mu_{k}}^{a}$ with $\mu_{1}, \ldots, \mu_{k} \leq \operatorname{dim} \gamma$, whereas the BV 2-form $\Omega_{\Sigma}$ couples the components $X_{\mu_{1} \cdots \mu_{k}}^{a}$ and $X_{v_{1} \cdots v_{l}}^{b}$ if and only if $\left\{\mu_{1}, \ldots, \mu_{k}\right\} \sqcup\left\{v_{1}, \ldots, \nu_{l}\right\}=\{1, \ldots, \operatorname{dim} \Sigma\}$ (for the sake of this argument we choose local Darboux coordinates on $\mathcal{M}$ in which $\omega_{a b}(x)$ does not depend on $x$ ). So, formally (61) is $\infty \cdot 0$, and we naively regularize it to zero. Thus, by Remark 5, we have (in the sense of our regularization) the classical master equation on $\mathcal{F}_{\Sigma} \times \mathcal{F}_{\gamma}$ :

$$
\left\{S_{\Sigma}+S_{\gamma}, S_{\Sigma}+S_{\gamma}\right\}_{\Omega_{\Sigma}+\Omega_{\gamma}}=0
$$

In the case when $\mathrm{i}: \gamma \rightarrow \Sigma$ is a diffeomorphism, we have

$$
\left\{S_{\gamma}, S_{\gamma}\right\}_{\Omega_{\Sigma}}=\left\{S_{\gamma}^{\text {target }}, S_{\gamma}^{\text {target }}\right\}_{\Omega_{\Sigma}}=(-1)^{\operatorname{dim} \Sigma} p^{*} \tau_{\gamma}^{\text {tot }}\left(\left\{\Theta^{\prime}, \Theta^{\prime}\right\}_{\omega}\right)
$$

Thus, (62) holds if and only if on the target we have

$$
\left\{\Theta^{\prime}, \Theta^{\prime}\right\}_{\omega}=0
$$

in addition to (30).

Remark 8. If $\operatorname{dim} \gamma=1, \omega^{\prime}$ is not automatically exact. The interesting case is when $\omega^{\prime}$ is not exact but satisfies Bohr-Sommerfeld integrality condition, see Remark 2. We assume that $\gamma$ is a circle; the discussion extends to a disjoint union of circles trivially. The kinetic part of the action for auxiliary fields $S_{\gamma}^{\mathrm{kin}}$ cannot be defined as in (54), because the primitive $\alpha^{\prime}$ does not exist globally on $\mathcal{N}$. Instead one can define

$$
\mathrm{e}^{i S_{\gamma}^{\mathrm{kin}(Y)}}:=\operatorname{Hol}_{Y_{(0)}^{*} \nabla^{\prime}}(\gamma) \in U(1)
$$

- the holonomy around $\gamma$ of the pull-back of the connection $\nabla^{\prime}$ (cf. Remark 2) by $Y_{(0)}: \gamma \rightarrow \mathcal{N}$. Here, the 1.h.s. should be viewed as one symbol. Target part of the action is defined as before, so we have

$$
\mathrm{e}^{i S_{\gamma}(X, Y)}=\mathrm{e}^{i \oint_{\gamma} \Theta^{\prime}\left(i^{*} X, Y\right)} \operatorname{Hol}_{Y_{(0)}^{*} \nabla^{\prime}}(\gamma)
$$

Otherwise, using Stokes' theorem one can rewrite the kinetic part of the auxiliary action as

$$
S_{\gamma}^{\operatorname{kin}}(Y)=\int_{D} \tilde{Y}_{(0)}^{*} \omega^{\prime}
$$

for $D$ a disk bounded by $\gamma$ and $\tilde{Y}_{(0)}$ an arbitrary extension of $Y_{(0)}$ from $\gamma$ to $D$. Due to integrality of $\omega^{\prime} / 2 \pi$, arbitrariness of $\tilde{Y}_{(0)}$ results in (65) being defined modulo multiples of $2 \pi$ :

$$
S_{\gamma}^{\operatorname{kin}}(Y) \in \mathbb{R} / 2 \pi \mathbb{Z}
$$

From this point of view, the 1.h.s. of (64) is well defined.

It is easy to see that the proof of Proposition 4 still works in this case (e.g., because one can find a primitive of $\omega^{\prime}$ in the tubular neighborhood of the loop $\left.Y_{(0)}(\gamma) \subset \mathcal{N}\right)$. 
Remark 9. Pre-observable (54) is invariant w.r.t. Diff $(\gamma)$ (reparametrizations) and $\operatorname{Diff}(\Sigma)$ (ambient diffeomorphisms) in the following sense. Let us denote explicitly the dependence of $S_{\gamma}$ on i: $\gamma \rightarrow \Sigma$ (through $p=\mathrm{i}^{*}$ in (54)) as $S_{\gamma}(X, Y$; i). Then for an ambient diffeomorphism $\Phi \in \operatorname{Diff}(\Sigma)$ and a reparametrization $\phi \in \operatorname{Diff}(\gamma)$ we have

$$
S_{\gamma}(X, Y ; \Phi \circ \mathrm{i} \circ \phi)=S_{\gamma}\left(\Phi^{*} X,\left(\phi^{-1}\right)^{*} Y ; \mathrm{i}\right)
$$

which can be seen directly from the definition (54).

\section{From Pre-observables to Observables}

The general idea of passage from pre-observables constructed in Section 5 to observables for the underlying AKSZ theory is by means of integrating out the auxiliary fields as in Proposition 1, i.e.,

$$
O_{\gamma}=\int_{\mathcal{L} \subset \mathcal{F}_{\gamma}} \mathrm{e}^{i S_{\gamma} \in C^{\infty}\left(\mathcal{F}_{\Sigma}\right)}
$$

where $\mathcal{L}$ is a Lagrangian submanifold of $\left(\mathcal{F}_{\gamma}, \Omega_{\gamma}\right)$. When we want to emphasize the dependence of $O_{\gamma}$ on the map i: $\gamma \rightarrow \Sigma$, we will write $O_{\gamma, \mathrm{i}}$.

Assuming that we can make sense of the path integral (67),

the observable $O_{\gamma}$ is expected to have the following properties:

(i) $O_{\gamma}$ depends on the fields of the ambient AKSZ theory only via pull-back by $\mathrm{i}: \gamma \rightarrow \Sigma$.

(ii) Gauge-invariance: $Q_{\Sigma} O_{\gamma}=0$ (which is, indeed, our definition of an observable).

(iii) The class of $O_{\gamma}$ in $Q_{\Sigma}$-cohomology is independent of deformations of the gauge fixing $\mathcal{L}$ (as a Lagrangian submanifold of $\mathcal{F}_{\gamma}$ ).

(iv) The class of $O_{\gamma}$ in $Q_{\Sigma}$-cohomology is invariant under isotopy of $\gamma$ (reparametrizations of $\gamma$ homotopic to identity): for $\phi \in \operatorname{Diff}_{0}(\gamma)$, we have

$$
O_{\gamma, \mathrm{i} \circ \phi}=O_{\gamma, \mathrm{i}}+Q_{\Sigma}(\cdots)
$$

(v) Invariance under ambient diffeomorphisms: for $\Phi \in \operatorname{Diff}(\Sigma)$, we have

$$
O_{\gamma, \Phi \circ \mathrm{i}}(X)=O_{\gamma, \mathrm{i}}\left(\Phi^{*} X\right)
$$

(vi) Invariance of the correlator $\left\langle O_{\gamma}\right\rangle$ under ambient isotopy: for $\Phi \in \operatorname{Diff}_{0}(\Sigma)$, we have

$$
\left\langle O_{\gamma, \Phi \mathrm{oi}}\right\rangle=\left\langle O_{\gamma, \mathrm{i}}\right\rangle
$$

Here, (i) follows from our construction of the pre-observable (54) and (v) follows from (i). Properties (ii) and (iii) are expected to hold in view of the Proposition 1 
for finite-dimensional fiber BV integrals. Property (iv) formally follows from (66), (iii) and $\operatorname{Diff}(\gamma)$-invariance of the measure on $\mathcal{F}_{\gamma}$ :

$$
\begin{aligned}
O_{\gamma, \text { io } \phi} & =\int_{\mathcal{L} \subset \mathcal{F}_{\gamma}} \mathcal{D} Y \mathrm{e}^{i S_{\gamma}(X, Y ; \text { i } \phi)}=\int_{\mathcal{L}} \mathcal{D} Y \mathrm{e}^{i S_{\gamma}\left(X,\left(\phi^{-1}\right)^{*} Y ; \mathrm{i}\right)} \\
& =\int_{\left(\phi^{-1}\right)^{*} \mathcal{L}}\left(\phi^{-1 *}\right)_{*}(\mathcal{D} Y) \mathrm{e}^{i S_{\gamma}(X, Y ; \mathrm{i})}=\int_{\left(\phi^{-1}\right)^{*} \mathcal{L}} \mathcal{D} Y \mathrm{e}^{i S_{\gamma}(X, Y ; \mathrm{i})} \\
& =\int_{\mathcal{L}} \mathcal{D} Y \mathrm{e}^{i S_{\gamma}(X, Y ; \mathrm{i})}+Q_{\Sigma}(\cdots)=O_{\gamma, \mathrm{i}}+Q_{\Sigma}(\cdots)
\end{aligned}
$$

Here, $\int_{\mathcal{L}} \mathcal{D} Y \cdots$ should be understood as $\left.\int_{\mathcal{L}} \sqrt{\mu}\right|_{\mathcal{L}} \cdots$ where $\mu$ is the path integral measure on $\mathcal{F}_{\gamma}$.

Note that we do use the fact that $\phi$ is an isotopy rather than a general diffeomorphism, since otherwise Lagrangians $\mathcal{L}$ and $\left(\phi^{-1}\right)^{*} \mathcal{L}$ are not guaranteed to be homotopic. Property (vi) formally follows from (v), Diff $(\Sigma)$-invariance of the AKSZ action (17) and of the path integral measure on $\mathcal{F}_{\Sigma}$, and from the BVStokes' theorem:

$$
\begin{aligned}
\left\langle O_{\gamma, \Phi \circ \mathrm{i}}\right\rangle & =\int_{\mathcal{L} \subset \mathcal{F}_{\Sigma}} \mathcal{D} X O_{\gamma, \Phi \circ \mathrm{i}}(X) \mathrm{e}^{i S_{\Sigma}(X)}=\int_{\mathcal{L}} \mathcal{D} X O_{\gamma, \mathrm{i}}\left(\Phi^{*} X\right) \mathrm{e}^{i S_{\Sigma}(X)} \\
& =\int_{\Phi^{*} \mathcal{L}}\left(\Phi^{*}\right)_{*}(\mathcal{D} X) O_{\gamma, \mathrm{i}}(X) \mathrm{e}^{i S_{\Sigma}\left(\left(\Phi^{-1}\right)^{*} X\right)}=\int_{\Phi^{*} \mathcal{L}} \mathcal{D} X O_{\gamma, \mathrm{i}}(X) \mathrm{e}^{i S_{\Sigma}(X)} \\
& =\int_{\mathcal{L}} \mathcal{D} X O_{\gamma, \mathrm{i}}(X) \mathrm{e}^{i S_{\Sigma}(X)}=\left\langle O_{\gamma, \mathrm{i}}\right\rangle
\end{aligned}
$$

Remark 10. For our construction of observable $O_{\gamma}$ it is not a priori necessary to impose any restrictions on the map $\mathrm{i}: \gamma \rightarrow \Sigma$. However, if we want to make sense of the correlator $\left\langle O_{\gamma}\right\rangle$ via perturbation theory for the path integral (which is outside of the scope of this paper), we have to require that $i$ is an embedding. The technical reason is that if $\mathrm{i}: \gamma \rightarrow \Sigma$ is not an embedding, it does not lift to a map between compactified configuration spaces of pairs of points on $\gamma$ and $\Sigma, \operatorname{Conf}_{2}(\gamma) \rightarrow$ $\operatorname{Conf}_{2}(\Sigma)$, which prevents one from defining the pull-back of the propagator of the ambient theory to $\gamma$, which in turn leads to Feynman diagrams for the correlator being ill-defined.

We will not try to give meaning to the path integral (67) in the most general situation here, but rather will discuss several special cases.

\subsection{CASE $\mathcal{N}=$ POINT}

In the case when $\mathcal{N}$ is a point, the target Hamiltonian $Q$-bundle is described in example (v) in Section 3.4: $\mathcal{A}=0, \omega^{\prime}=0, \Theta^{\prime}=\theta \in C^{\infty}(\mathcal{M})$ - a $Q$-cocycle of degree $p$ for the target $\mathcal{M}$. The associated pre-observable (54) for a $p$-dimensional closed manifold $\gamma$ and a map i: $\gamma \rightarrow \Sigma$ is: 


$$
\mathcal{F}_{\gamma}=\text { point }, \quad \mathcal{A}_{\gamma}=0, \quad \Omega_{\gamma}=0, \quad S_{\gamma}(X)=\int_{\gamma} \theta\left(i^{*} X\right)
$$

The passage to the observable (67) is trivial in this setting, since there are no auxiliary fields:

$$
O_{\gamma}=\mathrm{e}^{i \int_{\gamma} \theta\left(i^{*} X\right)}
$$

This observable obviously satisfies properties (i-v) above.

\subsection{CASE OF ONE-DIMENSIONAL OBSERVABLES}

Let $\mathrm{i}: \gamma=S^{1} \rightarrow \Sigma$ be a circle mapped into $\Sigma$ and suppose we have a Hamiltonian $Q$-bundle of degree 0 over the AKSZ target $(\mathcal{M}, Q, \omega=\delta \alpha, \Theta)$ with fiber data $\left(\mathcal{N}, \mathcal{A}, \omega^{\prime}=\delta \alpha^{\prime}, \Theta^{\prime}\right)$. We will assume for simplicity that $\mathcal{N}$ is concentrated in degree 0 , i.e., $\left(\mathcal{N}, \omega^{\prime}\right)$ is an ordinary (non-graded) symplectic manifold.

In local coordinates, the auxiliary superfield (60) is:

$$
Y^{i}(u, \mathrm{~d} u)=Y_{(0)}^{i}(u)+Y_{(1)}^{i}(u) \mathrm{d} u
$$

where $u$ is the coordinate on $\gamma=S^{1}$ and index $i$ corresponds to local coordinates on $\mathcal{N}$. For the pre-observable (54) we have

$$
\begin{aligned}
\mathcal{F}_{\gamma} & =\operatorname{Map}(T[1] \gamma, \mathcal{N}) \\
& =\left\{\left(Y_{(0)}, Y_{(1)}\right) \mid Y_{(0)}: \gamma \rightarrow \mathcal{N}, Y_{(1)} \in \Gamma\left(\gamma, T^{*} \gamma \otimes Y_{(0)}^{*} T \mathcal{N}\right)[-1]\right\}, \\
\Omega_{\gamma} & =-\oint_{\gamma} \omega_{i j}^{\prime}\left(Y_{(0)}\right) \delta Y_{(0)}^{i} \wedge \delta Y_{(1)}^{j}+\frac{1}{2} Y_{(1)}^{k} \partial_{k} \omega_{i j}^{\prime}\left(Y_{(0)}\right) \delta Y_{(0)}^{i} \wedge \delta Y_{(0)}^{j}, \\
S_{\gamma} & =\oint_{\gamma} \alpha_{i}^{\prime}\left(Y_{(0)}\right) \mathrm{d} Y_{(0)}^{i}+\Theta^{\prime}\left(i^{*} X, Y\right)
\end{aligned}
$$

One natural choice of Lagrangian in (67) is to set

$$
\mathcal{L}=\operatorname{Map}(\gamma, \mathcal{N}) \subset \operatorname{Map}(T[1] \gamma, \mathcal{N})
$$

i.e., the 1-form component $Y_{(1)}$ of the auxiliary superfield vanishes on $\mathcal{L}$. With this choice (67) becomes

$$
\begin{aligned}
O_{\gamma} & =\int_{\operatorname{Map}(\gamma, \mathcal{N})} \mathcal{D} Y_{(0)} \mathrm{e}^{i \oint_{\gamma} \alpha_{i}^{\prime}\left(Y_{(0)}\right) \mathrm{d} Y_{(0)}^{i}+\Theta^{\prime}\left(i^{*} X, Y_{(0)}\right)} \\
& =\int_{\operatorname{Map}(\gamma, \mathcal{N})} \mathcal{D} Y_{(0)} \mathrm{e}^{i \oint_{\gamma} \alpha_{i}^{\prime}\left(Y_{(0)}\right) \mathrm{d} Y_{(0)}^{i}+i^{*} X_{(1)}^{a} \partial_{a} \Theta^{\prime}\left(i^{*} X_{(0)}, Y_{(0)}\right)}
\end{aligned}
$$

Note that with this gauge fixing, one formally has a strict version of property (iv), namely that $O_{\gamma}$ is invariant under isotopy of $\gamma$ (instead of only the class of $O_{\gamma}$ in $Q_{\Sigma}$-cohomology being invariant). This follows from invariance of $\mathcal{L}$ under $\operatorname{Diff}(\gamma)$, together with $\operatorname{Diff}(\gamma)$-invariance of the path integral measure $\mathcal{D} Y_{(0)}$. 
The quantum mechanical path integral (73) can be understood in the Hamiltonian formalism, using the fiber geometric quantization of the target Hamiltonian $Q$-bundle, as follows.

PROPOSITION 5. Assume that the symplectic manifold $\left(\mathcal{N}, \omega^{\prime}\right)$ can be geometrically quantized to a complex vector space of states $\mathcal{H}$ and the Hamiltonian $\Theta^{\prime} \in$ $C^{\infty}(\mathcal{M} \times \mathcal{N})$ can be quantized (viewing coordinates on $\mathcal{M}$ as external parameters) to an operator-valued function on $\mathcal{M}, \hat{\Theta}^{\prime} \in C^{\infty}(\mathcal{M}) \otimes \operatorname{End}(\mathcal{H})$ satisfying

$$
Q \hat{\Theta}^{\prime}+i\left(\hat{\Theta}^{\prime}\right)^{2}=0
$$

(the quantum counterpart of the Eq. (30)). Then define $O_{\gamma}$ as the trace of a path ordered exponential ${ }^{2}$

$$
o_{\gamma}=\operatorname{tr}_{\mathcal{H}} \mathcal{P} \exp \left(i \oint_{\gamma} \hat{\Theta}^{\prime}\left(i^{*} X\right)\right)
$$

Then

$$
Q_{\Sigma} O_{\gamma}=0
$$

i.e., $O_{\gamma}$ is an observable.

Proof. Let $j:[0,1] \rightarrow \Sigma$ be a path in $\Sigma$ parameterized by $t \in[0,1]$. Let us denote $\psi=\hat{\Theta}^{\prime}\left(j^{*} X\right) \in \Omega^{\bullet}([0,1]) \hat{\otimes} C^{\infty}\left(\mathcal{F}_{\Sigma}\right) \otimes \operatorname{End}(\mathcal{H})$, also let $\psi_{(0)}(t)$ and $\psi_{(1)}(t, \mathrm{~d} t)$ be the 0 - and 1-form components of $\psi$. Consider the path ordered exponential

$$
\begin{aligned}
W_{j} & =\mathcal{P} \exp \left(i \int_{0}^{1} \psi_{(1)}\right) \\
& =\lim _{N \rightarrow \infty} \overleftarrow{\prod_{0 \leq k<N}}\left(\operatorname{id}_{\mathcal{H}}+i \iota_{\frac{1}{N} \partial_{t}} \psi_{(1)}(k / N, \mathrm{~d} t)\right) \in C^{\infty}\left(\mathcal{F}_{\Sigma}\right) \otimes \operatorname{End}(\mathcal{H})
\end{aligned}
$$

Then

$$
\begin{aligned}
Q_{\Sigma} W_{j}= & -i \int_{0}^{1} \mathcal{P} \exp \left(i \int_{t}^{1} \psi_{(1)}\right) \cdot Q_{\Sigma} \psi(t, \mathrm{~d} t) \cdot \mathcal{P} \exp \left(i \int_{0}^{t} \psi_{(1)}\right) \\
= & -i \int_{0}^{1} \mathcal{P} \exp \left(i \int_{t}^{1} \psi_{(1)}\right) \cdot\left(\mathrm{d} t \frac{\partial}{\partial t} \psi_{(0)}(t)-i\left[\psi_{(0)}(t), \psi_{(1)}(t, \mathrm{~d} t)\right]\right) \\
& \cdot \mathcal{P} \exp \left(i \int_{0}^{t} \psi_{(1)}\right) \\
= & -i \lim _{N \rightarrow \infty} \sum_{l=0}^{N-1} \overleftarrow{\prod_{l<k<N}}\left(\operatorname{id}_{\mathcal{H}}+i \iota_{\frac{1}{N} \partial_{t}} \psi_{(1)}\left(\frac{k}{N}, \mathrm{~d} t\right)\right) .
\end{aligned}
$$

${ }^{22}$ To define the path ordered exponential, we need to choose a starting point $p \in \gamma$, but the dependence on $p$ is canceled by taking the trace. 


$$
\begin{aligned}
& \cdot\left(\psi_{(0)}\left(\frac{l+1}{N}\right)\left(\operatorname{id}_{\mathcal{H}}+i \iota_{\frac{1}{N} \partial_{t}} \psi_{(1)}\left(\frac{l}{N}, \mathrm{~d} t\right)\right)\right. \\
- & \left.\left(\operatorname{id}_{\mathcal{H}}+i \iota_{\frac{1}{N} \partial_{t}} \psi_{(1)}\left(\frac{l}{N}, \mathrm{~d} t\right)\right) \psi_{(0)}\left(\frac{l}{N}\right)\right) . \\
& \cdot \prod_{0 \leq k<l}\left(\operatorname{id}_{\mathcal{H}}+i \iota_{\frac{1}{N} \partial_{t}} \psi_{(1)}\left(\frac{k}{N}, \mathrm{~d} t\right)\right) \\
= & -i\left(\psi_{(0)}(1) \cdot W_{j}-W_{j} \cdot \psi_{(0)}(0)\right)
\end{aligned}
$$

where we used that due to (74), we have $Q_{\Sigma} \psi=d \psi-\frac{i}{2}[\psi, \psi]$.

For $j=i: S^{1} \rightarrow \Sigma$, we have $j(0)=j(1)$ and applying (77), we get

$$
Q_{\Sigma} O_{\gamma}=\operatorname{tr}_{\mathcal{H}} Q_{\Sigma} W_{j}=-i \operatorname{tr}_{\mathcal{H}}\left[\hat{\Theta}^{\prime}\left(X_{(0)}(j(0))\right), W_{j}\right]=0
$$

Note that the construction of Proposition 5 does not require $\omega^{\prime}$ to be exact, it is enough to require that $\omega^{\prime}$ satisfies Bohr-Sommerfeld integrality condition.

\subsection{TORSION-LIKE OBSERVABLES}

Now let again $\mathrm{i}: \gamma \rightarrow \Sigma$ be an oriented closed manifold of arbitrary dimension mapped into $\Sigma$ and suppose that $\mathcal{N}$ is a finite-dimensional graded vector space, $\omega^{\prime}$ is a graded anti-symmetric pairing on $\mathcal{N}$ of degree $\operatorname{dim} \gamma-1$ and $\Theta^{\prime}$ is quadratic in $\mathcal{N}$ directions:

$$
\Theta^{\prime}(x, y)=\frac{1}{2} \omega^{\prime}(y, \theta(x) y)
$$

with $\theta \in C^{\infty}(\mathcal{M}) \otimes \operatorname{End}(\mathcal{N})$ of degree 1. Equation (30) is then equivalent to

$$
Q \theta-\theta^{2}=0
$$

We also assume that

$$
\operatorname{Str}_{\mathcal{N}} \theta(x)=0
$$

where $\operatorname{Str}_{\mathcal{N}}$ stands for the super-trace over $\mathcal{N}$. This assumption is necessary for the corresponding pre-observable to satisfy (42).

One way to make sense of the expression (67) is to choose a Riemannian metric on $\gamma$.

Then we have the Hodge decomposition for differential forms on $\gamma$ with values in $\mathcal{N}$

$$
\underbrace{\mathcal{N} \otimes \Omega^{\bullet}(\gamma)}_{\mathcal{F}_{\gamma}}=\underbrace{\mathcal{N} \otimes \Omega_{\text {harm }}^{\bullet}(\gamma)}_{\mathcal{F}_{\gamma}^{Z}} \oplus \underbrace{\mathcal{N} \otimes \Omega_{\text {exact }}^{\bullet}(\gamma) \oplus \overbrace{\mathcal{N} \otimes \Omega_{\text {co-exact }}^{\bullet}(\gamma)}^{\tilde{\mathcal{L}}}}_{\tilde{\mathcal{F}}_{\gamma}}
$$


which can be used to first take the BV integral over the complement of harmonic forms, as in Proposition 2, and then take the BV integral over the zero modes (harmonic forms):

$$
O_{\gamma}=\int_{\mathcal{L}_{\mathrm{Z}} \subset \mathcal{F}_{\gamma}^{z}} \mathcal{D} Y_{\mathrm{Z}} \int_{\tilde{\mathcal{L}} \subset \tilde{\mathcal{F}}_{\gamma}} \mathcal{D} \tilde{Y} \mathrm{e}^{\frac{i}{2} \int_{\gamma} \omega^{\prime}\left(Y, \mathrm{~d} Y+\theta\left(i^{*} X\right) Y\right)}
$$

where $Y=Y_{z}+\tilde{Y}$ is the splitting of the auxiliary superfield into the harmonic part and the part in the complement; $\mathcal{F}_{\gamma}^{Z}$ comes with the degree -1 symplectic structure $\Omega_{\gamma}^{\mathrm{Z}}$ induced from $\omega^{\prime}$ and the Poincare pairing on cohomology of $\gamma ; \mathcal{L}_{\mathrm{Z}} \subset \mathcal{F}_{\gamma}^{\mathrm{Z}}$ is a Lagrangian vector subspace w.r.t. $\Omega_{\gamma}^{z}$.

Remark 11. We call (81) a "torsion-like" observable, because in special cases (see Section 7.4) it is the Ray-Singer torsion of $\gamma$ equipped with a local system coming from the pull-back of the field of the ambient theory to $\gamma$. See, e.g., (109) where the evaluation of our observable for a circle embedded in a 3-manifold yields the standard formula for the Ray-Singer torsion of a circle endowed with a local system coming from the holonomy of the ambient connection. The idea of the path integral representation of Ray-Singer torsion goes back to Schwarz [17].

The internal integral in (81) is a path integral and can be made sense of using perturbation theory ${ }^{23}$ :

$$
\begin{aligned}
O_{\gamma}^{\mathrm{Z}}\left(X, Y_{\mathrm{Z}}\right)= & \exp \left(\frac{i}{2} \int_{\gamma} \omega^{\prime}\left(Y_{\mathrm{z}}, \theta\left(\mathrm{i}^{*} X\right)\left(\mathrm{id}+G \theta\left(\mathrm{i}^{*} X\right)\right)^{-1} Y_{\mathrm{Z}}\right)\right. \\
& \left.+\operatorname{Str}_{\mathcal{N}} \sum_{k \geq 2} \frac{(-1)^{k}}{2 k} \int_{\operatorname{Conf}_{k}(\gamma)} \overleftarrow{\prod_{1 \leq j \leq k}} \pi_{j+1, j}^{*} \eta \cdot \pi_{j}^{*} \theta\left(\mathrm{i}^{*} X\right)\right)
\end{aligned}
$$

The notations here are:

- $\operatorname{Conf}_{k}(\gamma)$ is the Fulton-Macpherson-Axelrod-Singer compactification of the configuration space of $k$ points on $\gamma$, cf. [4,5].

- $G=d^{*}\left(\Delta+\mathcal{P}_{\text {harm }}\right)^{-1}: \Omega^{\bullet}(\gamma) \rightarrow \Omega^{\bullet-1}(\gamma)$ is the Hodge-theoretic inverse of $d$; its integral kernel extends to a smooth $(\operatorname{dim} \gamma-1)$-form $\eta$ on $\operatorname{Conf}_{2}(\gamma)$ (the Hodge

\footnotetext{
${ }^{23} \mathrm{We}$ are working modulo constants here. We are also implicitly assuming convergence of the sum over $k$ in (82), which is an open condition on the field $X$. More precisely, we require that the component of $G \theta\left(i^{*} X\right)$ which is an $X$-dependent degree-preserving operator on $\mathcal{N} \otimes \Omega^{\bullet}(\gamma)$, has eigenvalues in the open unit disk on $\mathbb{C}$. Formula (82) is obtained by regarding $\frac{1}{2} \int_{\gamma} \omega^{\prime}(\tilde{Y}, d \tilde{Y})$ as the "free" part of the auxiliary action (which is non-degenerate on $\tilde{\mathcal{L}}$ and produces the propagator $-\eta$, cf., e.g., [14] $)$ and $\frac{1}{2} \int_{\gamma} \omega^{\prime}\left(Y_{\mathrm{Z}}, \theta Y_{\mathrm{Z}}\right)+\int_{\gamma} \omega^{\prime}\left(Y_{\mathrm{Z}}, \theta \tilde{Y}\right)+\frac{1}{2} \int_{\gamma} \omega^{\prime}(\tilde{Y}, \theta \tilde{Y})$ as a perturbation, producing 0 -, 1- and 2-valent vertices for the Feynman diagrams, respectively. Connected Feynman diagrams are: 0 -valent vertex, two 1-valent vertices connected by a sequence of $k \geq 02$-valent vertices (sum of these yields the first term in the exponential in (82)), and "polygons" - 1-loop diagrams containing $k \geq 2$ 2-valent vertices. The latter yield the last term in the exponential in (82).
} 
propagator), cf. $[4,5]$. By $\mathcal{P}_{\text {harm }}$ we denote the orthogonal projection to harmonic forms, using the Hodge inner product.

- $\pi_{j+1, j}: \operatorname{Conf}_{k} \rightarrow \operatorname{Conf}_{2}$ is the map associated to forgetting all points except points $j$ and $j+1$; also $\pi_{j}: \operatorname{Conf}_{k} \rightarrow \operatorname{Conf}_{1}=\gamma$ is forgetting all points except the point $j$. By convention, $\pi_{k+1, k}=\pi_{1, k}$.

- In the first term in the exponential in $(82), \theta\left(i^{*} X\right)$ is understood as a multiplication operator on $\Omega^{\bullet}(\gamma)$ and an endomorphism of $\mathcal{N}$, depending on ambient fields. Expression $\left(\mathrm{id}+G \theta\left(\mathrm{i}^{*} X\right)\right)^{-1}$ is understood as $\sum_{k=0}^{\infty}(-1)^{k}\left(G \theta\left(\mathrm{i}^{*} X\right)\right)^{k}$.

The BV integral over zero modes in (81) then is:

$$
\begin{aligned}
O_{\gamma}= & \int_{\mathcal{L}_{\mathrm{z}} \subset \mathcal{F}_{\gamma}^{\mathrm{Z}}} \mathrm{e}^{\frac{i}{2} \int_{\gamma} \omega^{\prime}\left(Y_{\mathrm{Z}}, \theta\left(i^{*} X\right)\left(\mathrm{id}+G \theta\left(\mathrm{i}^{*} X\right)\right)^{-1} Y_{\mathrm{Z}}\right)} \sqrt{\mu_{\mathrm{Z}}} \mathcal{L}_{\mathrm{z}} \cdot \\
& \cdot \exp \left(\operatorname{Str}_{\mathcal{N}} \sum_{k \geq 2} \frac{(-1)^{k}}{2 k} \int_{\operatorname{Conf}_{k}(\gamma)} \overleftarrow{\prod_{1 \leq j \leq k}} \pi_{j+1, j}^{*} \eta \cdot \pi_{j}^{*} \theta\left(i^{*} X\right)\right)
\end{aligned}
$$

where $\mu_{\mathrm{z}}$ is some fixed translation-invariant volume element on $\mathcal{F}_{\mathrm{z}}$.

Remark 12. The requirement that the integral over zero modes in (83) should converge imposes the following restriction on $\mathcal{L}_{\mathrm{z}}$. If $\left\{Y_{\mathcal{L}_{z}}^{I}\right\}$ are coordinates on $\mathcal{L}_{\mathrm{z}}$ and if we write

$$
\int_{\gamma} \omega^{\prime}\left(Y_{\mathrm{z}}, \theta\left(\mathrm{i}^{*} X\right)\left(\mathrm{id}+G \theta\left(\mathrm{i}^{*} X\right)\right)^{-1} Y_{\mathrm{z}}\right)=Y_{\mathcal{L}_{\mathrm{z}}}^{I} H_{I J}\left(\mathrm{i}^{*} X\right) Y_{\mathcal{L}_{\mathrm{z}}}^{J}
$$

then we require the even-even block (i.e., the block pairing coordinates $Y_{\mathcal{L}_{\mathrm{z}}}^{I}$ of even degree with coordinates $Y_{\mathcal{L}_{\mathrm{z}}}^{J}$ of even degree) of the matrix $\left(H_{I J}\right)$ to be invertible.

One universal way to satisfy this condition is to choose $\mathcal{L}_{\mathrm{z}} \subset \mathcal{F}_{\gamma}^{\mathrm{Z}}$ by setting all even coordinates on $\mathcal{F}_{\gamma}^{Z}$ to zero. With this choice, the integral over $\mathcal{L}_{\mathrm{z}}$ in (83) is (up to normalization) the Pfaffian of $\left(H_{I J}\right)$.

PROPOSITION 6. (i) Expression (83) is an observable, i.e.,

$$
Q_{\Sigma} O_{\gamma}=0
$$

(ii) Dependence of $O_{\gamma}$ on deformations of Riemannian metric on $\gamma$ and on deformations of $\mathcal{L}_{\mathrm{Z}}$ is $Q_{\Sigma}$ exact.

Proof. Define $S_{\gamma}^{\mathrm{Z}} \in C^{\infty}\left(\mathcal{F}_{\Sigma} \times \mathcal{F}_{\gamma}^{\mathrm{Z}}\right)$ by (82) and $O_{\gamma}^{\mathrm{z}}=\mathrm{e}^{i S_{\gamma}^{\mathrm{Z}}}$ :

$$
\begin{aligned}
S_{\gamma}^{\mathrm{Z}}= & \frac{1}{2} \int_{\gamma} \omega^{\prime}\left(Y_{\mathrm{Z}}, \theta(\mathrm{id}+G \theta)^{-1} Y_{\mathrm{Z}}\right) \\
& -i \operatorname{Str}_{\mathcal{N}} \sum_{k \geq 2} \frac{(-1)^{k}}{2 k} \int_{\operatorname{Conf}_{k}(\gamma)} \overleftarrow{\prod_{1 \leq j \leq k} \pi_{j+1, j}^{*} \eta \cdot \pi_{j}^{*} \theta}
\end{aligned}
$$

where $\theta$ actually means $\theta\left(i^{*} X\right)$. 
First let us check that $S_{\gamma}^{\mathrm{z}}$ is a semi-quantum pre-observable, i.e., satisfies the Eq. (42):

$$
Q_{\Sigma} S_{\gamma}^{\mathrm{z}}+\frac{1}{2}\left\{S_{\gamma}^{\mathrm{z}}, S_{\gamma}^{\mathrm{z}}\right\}_{\Omega_{\gamma}^{\mathrm{z}}}-i \Delta_{\mathrm{z}} S_{\gamma}^{\mathrm{z}}=0
$$

Indeed, we have

$$
\begin{aligned}
& \frac{1}{2}\left\{S_{\gamma}^{\mathrm{Z}}, S_{\gamma}^{\mathrm{Z}}\right\}_{\Omega_{\gamma}^{\mathrm{z}}}=\frac{1}{2} \int_{\gamma} \omega^{\prime}\left(Y_{\mathrm{z}},(\mathrm{id}+\theta G)^{-1} \theta \mathcal{P}_{\mathrm{harm}} \theta(\mathrm{id}+G \theta)^{-1} Y_{\mathrm{Z}}\right) \\
& -i \Delta_{\mathrm{Z}} S_{\gamma}^{\mathrm{Z}}=-\frac{i}{2} \operatorname{Str}_{\mathcal{F}_{\gamma}^{\mathrm{z}}}\left(\mathcal{P}_{\mathrm{harm}} \theta(\mathrm{id}+G \theta)^{-1}\right) \\
& Q_{\Sigma} S_{\gamma}^{\mathrm{Z}}=-\frac{1}{2} \int_{\gamma} \omega^{\prime}(Y_{\mathrm{z}},(\mathrm{id}+\theta G)^{-1} \underbrace{\left(d \theta+\theta^{2}\right)}_{Q_{\Sigma} \theta\left(\mathrm{i}^{*} X\right)}(\mathrm{id}+G \theta)^{-1} Y_{\mathrm{Z}}) \\
& -+\frac{i}{2} \operatorname{Str}_{\mathcal{N}} \sum_{k \geq 2}(-1)^{k} \int_{\operatorname{Conf}_{k}(\gamma)}\left(\overleftarrow{\prod_{2 \leq j \leq k}} \pi_{j+1, j}^{*} \eta \cdot \pi_{j}^{*} \theta\right) \cdot \pi_{2,1}^{*} \eta \cdot \pi_{1}^{*}\left(d \theta+\theta^{2}\right)
\end{aligned}
$$

Using Stokes' theorem, (88) can be written ${ }^{24}$ as

$$
\begin{aligned}
Q_{\Sigma} S_{\gamma}^{\mathrm{Z}}= & \frac{1}{2} \int_{\gamma} \omega^{\prime}\left(Y_{\mathrm{Z}},(\mathrm{id}+\theta G)^{-1} \theta([d, G]-\mathrm{id}) \theta(\mathrm{id}+G \theta)^{-1} Y_{\mathrm{Z}}\right) \\
& +\frac{i}{2} \operatorname{Str}_{\mathcal{N}} \sum_{k \geq 2}(-1)^{k+\operatorname{dim} \gamma} \int_{\operatorname{Conf}_{k}(\gamma)}\left(\overleftarrow{\prod_{2 \leq j \leq k}} \pi_{j+1, j}^{*} \eta \cdot \pi_{j}^{*} \theta\right) \cdot \pi_{2,1}^{*} d \eta \cdot \pi_{1}^{*} \theta \\
& +\frac{i}{2} \operatorname{Str}_{\mathcal{N}} \sum_{k \geq 2}(-1)^{k} \int_{\operatorname{Conf}_{k}(\gamma)}\left(\overleftarrow{\prod_{2 \leq j \leq k}} \pi_{j+1, j}^{*} \eta \cdot \pi_{j}^{*} \theta\right) \cdot \pi_{2,1}^{*} \eta \cdot \pi_{1}^{*} \theta^{2} \\
& -\frac{i}{2} \operatorname{Str}_{\mathcal{N}} \sum_{k \geq 2} \frac{(-1)^{k+k \operatorname{dim} \gamma}}{k} \int_{\partial \operatorname{Conf}_{k}(\gamma)} \overleftarrow{\prod_{1 \leq j \leq k} \pi_{j+1, j}^{*} \eta \cdot \pi_{j}^{*} \theta}
\end{aligned}
$$

Two last terms here cancel due to the contribution of principal boundary strata of $\operatorname{Conf}_{k}(\gamma)$, where points $j$ and $j+1$ collapse (and we use the property of the propagator that the fiber integral of $\eta$ over fibers of $\partial \operatorname{Conf}_{2}(\gamma) \rightarrow \gamma$ is the constant function $\left.-(-1)^{\operatorname{dim} \gamma} \mathbf{1} \in C^{\infty}(\gamma)\right)$. Hidden boundary strata (i.e., those corresponding to the collapse of $\geq 3$ points) do not contribute due to the fact that the collapsed

\footnotetext{
${ }^{24}$ The first term in (89) appears for the following reason. We calculate $\left[d,(\mathrm{id}+\theta G)^{-1} \theta\right]=-(\mathrm{id}+$ $\theta G)^{-1} \underbrace{[d, \theta G]}_{d \theta G-\theta[d, G]} \underbrace{(\mathrm{id}+\theta G)^{-1} \theta}_{\theta(\mathrm{id}+G \theta)^{-1}}+(\mathrm{id}+\theta G)^{-1} d \theta=(\mathrm{id}+\theta G)^{-1} d \theta(\mathrm{id}+G \theta)^{-1}+(\mathrm{id}+\theta G)^{-1} \theta[d, G] \theta(\mathrm{id}+$ $G \theta)^{-1}$. This implies, since $d$ appearing in the 1.h.s. annihilates the zero modes, that in the expression $\omega^{\prime}\left(Y_{\mathrm{Z}},(\mathrm{id}+\theta G)^{-1} d \theta(\mathrm{id}+G \theta)^{-1} Y_{\mathrm{Z}}\right)$ one can replace $d \theta$ with $-\theta[d, G] \theta$.
} 
subgraph of the "wheel" graph either has a vertex of valence 2, and then the integral vanishes by (anti-) symmetry of $\eta$ on $\partial \operatorname{Conf}_{2}(\gamma)$ w.r.t. the antipodal involution, or the collapsed subgraph has an isolated vertex, and then the integral vanishes for degree reasons, cf. [7].

Since $[d, G]=$ id $-\mathcal{P}_{\text {harm }}$ and since $d \eta=-(-1)^{\operatorname{dim} \gamma} \mathbf{P}_{\text {harm }}$ where $\mathbf{P}_{\text {harm }} \in$ $\Omega^{\operatorname{dim} \gamma}(\gamma \times \gamma)$ is the integral kernel of $\mathcal{P}_{\text {harm }}$, collecting $(86,87,89)$ we get the semi-quantum master equation (85).

Next, knowing that (85) holds, we infer by Proposition 1 that $O_{\gamma}=\int_{\mathcal{L}_{z} \subset \mathcal{F}_{\gamma}^{z}} \mathrm{e}^{i S_{\gamma}^{z}}$ given by (83) satisfies $Q_{\Sigma} O_{\gamma}=0$. Thus we proved (i).

To prove (ii), we first note that deformations of $\mathcal{L}_{\mathrm{z}}$ induce $Q_{\Sigma}$-exact shifts in $O_{\gamma}$ by Proposition 1.

Next, we check the dependence of $S_{\gamma}^{z}$ on metric on $\gamma$.

Let us now be more careful with the space of zero modes and define it as $\mathcal{F}_{\gamma}^{z}=$ $\mathcal{N} \otimes H^{\bullet}(\gamma)$-the $\mathcal{N}$-valued de Rham cohomology of $\gamma$; it comes with a metricdependent embedding of cohomology into differential forms $i: H^{\bullet}(\gamma) \hookrightarrow \Omega^{\bullet}(\gamma)$ which realizes cohomology classes by harmonic forms. In view of this redefinition we should now write $i\left(Y_{\mathrm{Z}}\right)$ instead of $Y_{\mathrm{Z}}$ in (84).

Now assume that we have a path in the space of Riemannian metrics on $\gamma$, $g_{t} \in \operatorname{Met}(\gamma)$ with $t \in[0,1]$ the parameter. Then we have the $t$-dependent Hodgetheoretic inverse $G_{t}$ for de Rham operator, the $t$-dependent propagator $\eta_{t}$ and the $t$-dependent embedding of cohomology into differential forms $i_{t}$ with exact timederivatives:

$$
\frac{\mathrm{d}}{\mathrm{d} t} G_{t}=\left[d, H_{t}\right], \quad \frac{\partial}{\partial t} \eta_{t}=d \zeta_{t}, \quad \frac{\partial}{\partial t} i_{t}=d j_{t}
$$

for some linear operator $H_{t}: \Omega^{\bullet}(\gamma) \rightarrow \Omega^{\bullet-2}(\gamma)$ with smooth integral kernel $\zeta_{t} \in$ $\Omega^{\operatorname{dim} \gamma-2}(\gamma \times \gamma)$ and some $j_{t}: H^{\bullet}(\gamma) \rightarrow \Omega^{\bullet-1}(\gamma) ; H_{t}, \zeta_{t}$ and $j_{t}$ depend on $g_{t}$ and $\frac{\partial}{\partial t} g_{t}$. Then, one can introduce the "extended" versions of $G, \eta$ and $i$ :

$$
\begin{aligned}
\tilde{G} & =G_{t}+\mathrm{d} t H_{t} \in \Omega^{\bullet}([0,1]) \hat{\otimes} \operatorname{End}\left(\Omega^{\bullet}(\gamma)\right) \\
\tilde{\eta} & =\eta_{t}+\mathrm{d} t \zeta_{t} \in \Omega^{\operatorname{dim} \Sigma-1}\left([0,1] \times \operatorname{Conf}_{2}(\gamma)\right) \\
\tilde{i} & =i_{t}+\mathrm{d} t j_{t} \in \Omega^{\bullet}([0,1]) \hat{\otimes} \operatorname{Hom}\left(H^{\bullet}(\gamma), \Omega^{\bullet}(\gamma)\right)
\end{aligned}
$$

They satisfy respectively

$$
\begin{aligned}
\left(\mathrm{d} t \frac{\partial}{\partial t}+\left[d_{\gamma}, \bullet\right]\right) \tilde{G} & =\mathrm{id}-\mathcal{P}_{\text {harm }} \\
\left(\mathrm{d} t \frac{\partial}{\partial t}+d_{\operatorname{Conf}_{2}(\gamma)}\right) \tilde{\eta} & =-(-1)^{\operatorname{dim} \gamma} \mathbf{P}_{\text {harm }} \\
\left(\mathrm{d} t \frac{\partial}{\partial t}+d_{\gamma}\right) \tilde{i} & =0
\end{aligned}
$$

Next, we define the extended version of $S_{\gamma}^{z}, \tilde{S}_{\gamma}^{\mathrm{z}} \in \Omega^{\bullet}([0,1]) \hat{\otimes} C^{\infty}\left(\mathcal{F}_{\Sigma} \times \mathcal{F}_{\gamma}^{\mathrm{Z}}\right)$ by substituting $\tilde{G}$ instead of $G, \tilde{\eta}$ instead of $\eta$ and $\tilde{i}\left(Y_{\mathrm{Z}}\right)$ instead of $Y_{\mathrm{Z}}$ into (84). Repeating 
our proof of (85) in the extended case we find that

$$
\left(\mathrm{d} t \frac{\partial}{\partial t}+Q_{\Sigma}\right) \tilde{S}_{\gamma}^{\mathrm{z}}+\frac{1}{2}\left\{\tilde{S}_{\gamma}^{\mathrm{z}}, \tilde{S}_{\gamma}^{\mathrm{z}}\right\}_{\Omega_{\gamma}^{z}}-i \Delta_{\mathrm{z}} \tilde{S}_{\gamma}^{\mathrm{z}}=0
$$

which, when we decompose $\tilde{S}_{\gamma}^{z}$ into the 0 -form and 1 -form parts w.r.t. $t$, $\tilde{S}_{\gamma}^{z}=$ $S_{\gamma}^{\mathrm{Z}}(t)+\mathrm{d} t R_{\gamma}^{\mathrm{Z}}(t)$, is equivalent to semi-quantum master equation (85) for $S_{\gamma}^{\mathrm{Z}}(t)$ plus the equation

$$
\frac{\partial}{\partial t} S_{\gamma}^{\mathrm{Z}}(t)=Q_{\Sigma} R_{\gamma}^{\mathrm{Z}}(t)+\left\{S_{\gamma}^{\mathrm{Z}}(t), R_{\gamma}^{\mathrm{Z}}(t)\right\}_{\Omega_{\gamma}^{\mathrm{z}}}-i \Delta_{\mathrm{z}} R_{\gamma}^{\mathrm{Z}}(t)
$$

Therefore (cf. (45)), semi-quantum pre-observables $S_{\gamma}^{\mathrm{Z}}(t)$ are equivalent for all values of the parameter $t$ and so by item (iii) of Proposition 1, dependence of the induced observable $O_{\gamma}(t)=\int_{\mathcal{L}_{\mathrm{z}} \subset \mathcal{F}_{\gamma}^{\mathrm{z}}} \mathrm{e}^{i S_{\gamma}^{\mathrm{Z}}(t)}$ on $t$ is $Q_{\Sigma}$-exact. This finishes the proof of (ii).

\section{Examples of Observables}

Here, we will illustrate the construction of AKSZ pre-observables/observables of Sects. 5,6 by several explicit examples.

\subsection{OBSERVABLES WITH $\mathcal{N}$ A POINT}

First, we give some examples of the situation described in Section 6.1.

- For abelian Chern-Simons theory (22) we may take $\theta=c \psi \in C^{\infty}(\mathbb{R}[1])$ with $c \in$ $\mathbb{R}$ any constant. This yields by (70) a one-dimensional observable for $\mathrm{i}: \gamma=S^{1} \hookrightarrow$ $\Sigma$

$$
O_{\gamma}=\mathrm{e}^{i c \oint_{\gamma} i^{*} A_{(1)}}
$$

- the abelian Wilson loop; parameter $c$ can be viewed as the weight of a onedimensional representation of the gauge group $\mathbb{R}$. Note that this is also an observable for the abelian $B F$ theory (26).

- For $D$-dimensional abelian $B F$ theory, take $\theta=c \xi$ with $c \in \mathbb{R}$ a parameter. The associated observable for a closed oriented submanifold $\mathrm{i}: \gamma \hookrightarrow \Sigma$ of codimension 2 is

$$
O_{\gamma}=\mathrm{e}^{i c \int_{\gamma} i^{*} B_{(D-2)}}
$$

- For two-dimensional $B F$ theory assume that $\mathfrak{g}$ is quadratic so that we can identify $\mathfrak{g}^{*}$ with $\mathfrak{g}$ and let $\rho: \mathfrak{g} \rightarrow \operatorname{End}(R)$ be a representation. Set $\theta=$ $-i \log \operatorname{tr}_{R} f(\rho(\xi))$ for $f \in C^{\infty}(\mathbb{R})$ a function. The corresponding zero-dimensional observable for $\gamma \in \Sigma$ a point is

$$
O_{\gamma}=\operatorname{tr}_{R} f\left(\rho\left(\left.B_{(0)}\right|_{\gamma}\right)\right)
$$


- For two-dimensional abelian $B F$ theory take $\theta=f(\xi) \psi$ for $f \in C^{\infty}(\mathbb{R})$. The corresponding one-dimensional observable for $\mathrm{i}: \gamma=S^{1} \hookrightarrow \Sigma$ is

$$
O_{\gamma}=\mathrm{e}^{i \oint_{\gamma} i^{*}(A \cdot f(B))}
$$

Note that for $f$ non-constant, $O_{\gamma}$ depends non-trivially on components of the superfields of ghost number $\neq 0$, namely on $A_{(0)}$ and $B_{(1)}$ :

$$
O_{\gamma}=\mathrm{e}^{i \oint_{\gamma} i^{*}\left(A_{(1)} \cdot f\left(B_{(0)}\right)+A_{(0)} \cdot B_{(1)} \cdot f^{\prime}\left(B_{(0)}\right)\right)}
$$

- For Poisson sigma model and $\theta=f \in C^{\infty}(M)$ a Casimir of the Poisson structure (i.e., $[\pi, f]=0$, with [,] the Schouten-Nijenhuis bracket on polyvector fields on $M)$, we get a zero-dimensional observable for $\gamma$ a point in $\Sigma$ :

$$
O_{\gamma}=\mathrm{e}^{i f\left(\left.X_{(0)}\right|_{\gamma}\right)}
$$

- For Poisson sigma model and $v \in \mathfrak{X}(M)$ a Poisson vector field (i.e., $[\pi, v]=0$ ), set $\theta=\langle p, v(x)\rangle$. The associated one-dimensional observable is:

$$
O_{\gamma}=\mathrm{e}^{i \oint_{\gamma} i^{*}\langle\eta, v(X)\rangle}
$$

Note that if $\tilde{v}=v+[\pi, f]$-another Poisson vector field giving the same class in Poisson cohomology of $M$ as $v$, then the observable $\tilde{O}_{\gamma}$ constructed from $\tilde{v}$ by (95) is equivalent to $O_{\gamma}:\langle p, \tilde{v}(x)\rangle-\langle p, v(x)\rangle=Q f(x)$ on the target implies $\oint_{\gamma} \mathrm{i}^{*}\langle\eta, \tilde{v}(X)\rangle-\oint_{\gamma} \mathrm{i}^{*}\langle\eta, v(X)\rangle=-Q_{\Sigma} \oint_{\gamma} \mathrm{i}^{*} f(X)$ on the mapping space $\mathcal{F}_{\Sigma}$. This implies in turn

$$
\tilde{O}_{\gamma}=O_{\gamma}+Q_{\Sigma}\left(-i \int_{0}^{1} \mathrm{~d} t \mathrm{e}^{i \oint_{\gamma} i^{*}\langle\eta,(1-t) v(X)+t \tilde{v}(X)\rangle} \oint_{\gamma} \mathrm{i}^{*} f(X)\right)
$$

Thus (95) defines a map from Poisson cohomology of $M$ in degree one to $Q_{\Sigma^{-}}$ cohomology in degree zero.

Note that the observable (92) is a special case of (94) when we take $M$ to be $\mathfrak{g}^{*}$ with Kirillov-Kostant Poisson structure. Also, (93) is a special case of (95) for $M=\mathbb{R}$ with $\pi=0$.

\subsection{WILSON LOOP OBSERVABLE IN CHERN-SIMONS THEORY}

Here, we will combine the discussion of Section 6.2 with the example (iv) of Section 3.4.

Let $\mathfrak{g}$ be a quadratic Lie algebra and let $\left(M, \omega_{M}\right)$ be a symplectic manifold with a Hamiltonian action of $\mathfrak{g}$ with equivariant moment map $\mu: M \rightarrow \mathfrak{g}^{*}$. Further, we assume that $\omega_{M}$ satisfies Bohr-Sommerfeld integrality condition and is the curvature of a $U(1)$-connection $\nabla$ on a line bundle $L$ over $M$.

Then, we have a degree 0 Hamiltonian $Q$-bundle structure on $\mathfrak{g}[1] \times M \rightarrow \mathfrak{g}[1]$, with base structure given by (20) and fiber structure (39), with the correction that 
now we relaxed the exactness condition on $\omega_{M}$. Applying the construction of Section 5 to this target data we get the following one-dimensional pre-observable for Chern-Simons theory:

$$
\begin{aligned}
\mathcal{F}_{\gamma} & =\operatorname{Map}(T[1] \gamma, M) \\
& =\left\{\left(Y_{(0)}, Y_{(1)}\right) \mid Y_{(0)}: \gamma \rightarrow M, Y_{(1)} \in \Gamma\left(\gamma, T^{*} \gamma \otimes Y_{(0)}^{*} T M\right)[-1]\right\} \\
\Omega_{\gamma} & =-\oint_{\gamma} \omega_{M}(Y)=-\oint_{\gamma} \omega_{i j}\left(Y_{(0)}\right) \delta Y_{(0)}^{i} \wedge \delta Y_{(1)}^{j}+\frac{1}{2} Y_{(1)}^{k} \partial_{k} \omega_{i j}\left(Y_{(0)}\right) \delta Y_{(0)}^{i} \wedge \delta Y_{(0)}^{j}, \\
\mathrm{e}^{i S_{\gamma}} & =\operatorname{Hol}_{Y_{(0)}^{*}} \nabla(\gamma) \mathrm{e}^{i \oint_{\gamma}\left\langle i^{*} A, \mu(Y)\right\rangle}=\operatorname{Hol}_{Y_{(0)}^{*}}^{*}(\gamma) \mathrm{e}^{i \oint_{\gamma}\left\langle i^{*} A_{(1)}, \mu\left(Y_{(0)}\right)\right\rangle+\left\langle i^{*} A_{(0)}, Y_{(1)}^{i} \partial_{i} \mu\left(Y_{(0)}\right)\right\rangle}
\end{aligned}
$$

where $\mathrm{i}: \gamma=S^{1} \hookrightarrow \Sigma$ is a circle embedded into 3-manifold $\Sigma, A=\sum_{k=0}^{3} A_{(k)}$ is the superfield of the ambient Chern-Simons theory, $Y=Y_{(0)}+Y_{(1)}$ is the auxiliary superfield (21), with components $Y_{(0)}, Y_{(1)}$ having internal degrees 0 and -1 , respectively; indices $i, j, k$ refer to local coordinates on $M$.

To construct an observable out of the pre-observable (96), we impose the gauge fixing (72) which consists in setting $Y_{(1)}=0$. The path integral (73) reads

$$
O_{\gamma}=\int_{\operatorname{Map}(\gamma, M)} \mathcal{D} Y_{(0)} \operatorname{Hol}_{Y_{(0)}^{*} \nabla}(\gamma) \mathrm{e}^{i \oint_{\gamma}\left\langle i^{*} A_{(1)}, \mu\left(Y_{(0)}\right)\right\rangle}
$$

Assume that there exists a Lagrangian polarization $P$ of $\left(M, \omega_{M}\right)$ such that the geometric quantization of $(M, \omega, L, \nabla, P)$ yields a space of states $\mathcal{H}$ and components $\mu_{a} \in C^{\infty}(M)$ of the moment map are quantized into operators $\hat{\mu}_{a} \in \operatorname{End}(\mathcal{H})$ such that the map $\hat{\mu}: \mathfrak{g} \rightarrow \operatorname{End}(\mathcal{H})$ sending generator $T_{a} \in \mathfrak{g}$ into $\hat{\mu}_{a}$ is a Lie algebra homomorphism (i.e., $\hat{\mu}$ is a representation of $\mathfrak{g}$ on $\mathcal{H}$ ). Then

$$
\hat{\Theta}^{\prime}=-i\langle\psi, \hat{\mu}\rangle \in C^{\infty}(\mathfrak{g}[1]) \otimes \operatorname{End}(\mathcal{H})
$$

(where we view $\hat{\mu}$ as an element of $\mathfrak{g}^{*} \otimes \operatorname{End}(\mathcal{H})$ ) is the quantization of $\Theta^{\prime}=$ $\langle\psi, \mu(x)\rangle$ satisfying (74). Then the construction of Proposition 5 produces out of the pre-observable (96) the usual Wilson loop of the connection component $A_{(1)}$ of the ambient superfield in representation $\hat{\mu}$ along $\gamma$ :

$$
O_{\gamma}=\operatorname{tr}_{\mathcal{H}} \mathcal{P} \exp \oint_{\gamma} \hat{\mu}\left(i^{*} A_{(1)}\right)
$$

which is indeed a gauge-invariant observable (i.e. $Q_{\Sigma} O_{\gamma}=0$ ) for Chern-Simons theory on $\Sigma$.

Expression (98) can be viewed as a regularization of the path integral (97).

Coadjoint orbit case. In case when $\left(M, \omega_{M}\right)$ is an integral coadjoint orbit of a compact Lie group $G$ such that $\mathfrak{g}=\operatorname{Lie}(G)$, with $\omega_{M}$ the Kirillov symplectic structure and $\mu: M \hookrightarrow \mathfrak{g}^{*}$ the embedding of $M$ into $\mathfrak{g}^{*}$ (which corresponds to the 
coadjoint Hamiltonian action of $G$ on $M$ ), formula (97) becomes the AlekseevFaddeev-Shatashvili path integral formula for the Wilson loop [2]. ${ }^{25}$

The respective $\hat{\mu}$ is the irreducible representation of $G$ associated by Kirillov's orbit method to the coadjoint orbit $M$, and (98) is just the Wilson loop in this irreducible representation.

\section{3. "WILSON LOOPS" IN POISSON SIGMA MODEL}

Let $(M, \pi)$ be a Poisson manifold, $\left(N, \omega_{N}=\delta \alpha_{N}\right)$ an exact symplectic manifold and $F: N \rightarrow \mathfrak{X}(M)$ a map ${ }^{26}$ with the property

$$
\frac{1}{2}\{F, F\}_{\omega_{N}}+[\pi, F]=0
$$

where [,] is the Schouten-Nijenhuis bracket on polyvector fields on $M$; expression $\frac{1}{2}\{F, F\}$ is a map from $N$ to bivectors on $M$, given in local coordinates as $\frac{1}{2}\left(\omega^{-1}(y)\right)^{a b} \frac{\partial}{\partial y^{a}} F^{i}(x, y) \frac{\partial}{\partial y^{b}} F^{j}(x, y) \frac{\partial}{\partial x^{i}} \wedge \frac{\partial}{\partial x^{j}}$ where $\left\{x^{i}\right\}$ are local coordinates on $M$ and $\left\{y^{a}\right\}$ are local coordinates on $N$. Then we have a degree 0 Hamiltonian $Q$-bundle structure on $T^{*}[1] M \times N \rightarrow T^{*}[1] M$ with base structure (27) and fiber structure

$$
\begin{aligned}
& \mathcal{N}=N, \quad \mathcal{A}=\left\langle p,\{F, \bullet\}_{\omega_{N}}\right\rangle, \\
& \omega^{\prime}=\omega_{N}, \quad \alpha^{\prime}=\alpha_{N}, \quad \Theta^{\prime}=\langle p, F\rangle \in C^{\infty}\left(T^{*}[1] M \times N\right)
\end{aligned}
$$

The corresponding one-dimensional pre-observable for Poisson sigma model associated to $(M, \pi)$ is given by $\mathcal{F}_{\gamma}, \Omega_{\gamma}$ as in (96), changing $M$ to $N$, and the auxiliary action is

$$
S_{\gamma}=\oint_{\gamma} \alpha_{a}(Y) \mathrm{d} Y^{a}+\left\langle i^{*} \eta, F\left(\mathrm{i}^{*} X, Y\right)\right\rangle
$$

${ }^{25}$ For reader's convenience, we recall the main result of [2], Let $A \in \Omega^{1}\left(S^{1}, \mathfrak{g}\right)$ be a connection 1 -form for a principal connection in a $G$-bundle over a circle. We fix $\lambda \in \mathfrak{g}^{*}$ such that the coadjoint orbit $o_{\lambda}=\left\{g \lambda g^{-1} \mid g \in G\right\} \subset \mathfrak{g}^{*}$ passing through $\lambda$ is integral (for convenience we denote $g \lambda g^{-1}:=$ $\operatorname{Ad}_{g}^{*} \lambda$ ). Denote by $\rho$ the irreducible unitary representation of $G$ corresponding to $o_{\lambda}$ by Kirillov's orbit method; denote the representation space $R$. Then by [2] we have that $\operatorname{tr}_{R} \mathcal{P} \exp \oint \rho(A)=$ $\int \mathcal{D} g \mathrm{e}^{i S_{A F S}(A, g)}$ where the 1.h.s. is the Wilson loop in representation $\rho$, the r.h.s. is the path integral over maps $g: S^{1} \rightarrow G$; the action in the r.h.s. is $S_{A F S}(A, g)=\oint\left\langle\lambda, g^{-1} d g+g^{-1} A g\right\rangle$. This action is preserved by ultralocal gauge transformations $g \mapsto g h$ with $h: S^{1} \rightarrow \operatorname{Stab}_{\lambda}$, where $\operatorname{Stab}_{\lambda} \subset G$ consists of elements commuting with $\lambda$. One can pass to the reduction by this symmetry, introducing a new variable $b=g \lambda g^{-1} \in o_{\lambda} \simeq G / \operatorname{Stab}_{\lambda}$. We have $\mathrm{e}^{i S_{A F S}(A, g)}=\mathrm{Hol}_{\nabla}(\operatorname{im}(b)) \cdot \mathrm{e}^{i \oint\langle b, A\rangle}$ where $\nabla$ is the connection in the prequantum line bundle $L$ over $o_{\lambda}$. (Note that the prequantum line bundle $(L, \nabla)$ on $o_{\lambda}$ can be constructed as the push-forward of the trivial circle bundle on $G$ with connection given by 1 -form $\alpha=\left\langle\lambda, g^{-1} \delta g\right\rangle \in \Omega^{1}(G)$ with respect to presymplectic reduction by the kernel of $\delta \alpha$. Integrality of $o_{\lambda}$ is necessary and sufficient for this push-forward to exist.) To go back to our notations, we should set $M=o_{\lambda}, Y_{(0)}=b, i^{*} A_{(1)}=A, \hat{\mu}=\rho, \mathcal{H}=R$.

${ }^{26}$ Alternatively, one can view $F$ as a vertical vector field on the trivial fiber bundle $N \times M \rightarrow N$. 
The corresponding observable is formally given (in the gauge (72)) by the path integral (73):

$$
O_{\gamma}=\int_{\operatorname{Map}(\gamma, N)} \mathcal{D} Y_{(0)} \mathrm{e}^{i \oint_{\gamma} \alpha_{a}\left(Y_{(0)}\right) \mathrm{d} Y_{(0)}^{a}+\left\langle i^{*} \eta, F\left(i^{*} X, Y_{(0)}\right)\right\rangle}
$$

Assume that $\left(N, \omega_{N}\right)$ can be geometrically quantized to a space of states $\mathcal{H}$ and $F$ is quantized to an operator-valued vector field $\hat{F} \in \operatorname{End}(\mathcal{H}) \hat{\otimes} \mathfrak{X}(M)$ satisfying

$$
[\pi, \hat{F}]+i \hat{F} \wedge \hat{F}=0
$$

Then (75) gives

$$
O_{\gamma}=\operatorname{tr}_{\mathcal{H}} \mathcal{P} \exp \left(i \oint_{\gamma}\left\langle i^{*} \eta, \hat{F}\left(i^{*} X\right)\right\rangle\right)
$$

which can be regarded as the evaluation of the path integral (101) via Hamiltonian formalism. By Proposition 5, $O_{\gamma}$ is indeed an observable for the Poisson sigma model.

Remark 13. Note that in case $N=$ point we get back the observable (95). Also, in case $M=\mathfrak{g}^{*}$ with Kirillov-Kostant Poisson structure and requiring that $F$ takes values in constant vector fields on $F$, Eq. (99) becomes the equation on equivariant moment map and the corresponding observable (103) is the usual Wilson loop (98) for 2-dimensional $B F$ theory.

\subsection{TORSION OBSERVABLES IN CHERN-SIMONS THEORY}

Fix $n^{\prime} \in\{-1,0,1,2\}$ and $m \in \mathbb{Z}$ (only the parity of $m$ will matter for our discussion). Let again $\mathfrak{g}$ be a quadratic Lie algebra and let $\rho: \mathfrak{g} \rightarrow \operatorname{End}(R)$ be a representation on a vector space $R$ with values in traceless matrices (cf. (79)). Then we have a degree $n^{\prime}$ Hamiltonian $Q$-bundle structure on $\mathfrak{g}[1] \oplus R[m] \oplus R^{*}\left[n^{\prime}-m\right] \rightarrow \mathfrak{g}[1]$ with base structure (20) and fiber structure

$$
\begin{aligned}
& \mathcal{N}=R[m] \oplus R^{*}\left[n^{\prime}-m\right], \quad \mathcal{A}=\left\langle\rho(\psi) q, \frac{\partial}{\partial q}\right\rangle+\left\langle\rho^{*}(\psi) p, \frac{\partial}{\partial p}\right\rangle, \\
& \omega^{\prime}=\langle\delta p, \delta q\rangle, \quad \alpha^{\prime}=\langle p, \delta q\rangle, \quad \Theta^{\prime}=\langle p, \rho(\psi) q\rangle
\end{aligned}
$$

where $\rho^{*}: \mathfrak{g} \rightarrow \operatorname{End}\left(R^{*}\right)$ is the representation dual to $\rho ; q$ is the $R$-valued coordinate of degree $m$ on $R[m]$ and $p$ is the $R^{*}$-valued coordinate of degree $n^{\prime}-m$ on $R^{*}\left[n^{\prime}-m\right]$.

The corresponding pre-observable for Chern-Simons theory on a closed oriented 3-manifold $\Sigma$, for $\mathrm{i}: \gamma \hookrightarrow \Sigma$ an embedded closed oriented $\left(n^{\prime}+1\right)$-manifold, reads: 


$$
\begin{aligned}
& \mathcal{F}_{\gamma}=R[m] \otimes \Omega^{\bullet}(\gamma) \oplus R^{*}\left[n^{\prime}-m\right] \otimes \Omega^{\bullet}(\gamma), \quad \Omega_{\gamma}=\int_{\gamma}\langle\delta \mathbf{p}, \delta \mathbf{q}\rangle, \\
& S_{\gamma}=\int_{\gamma}\langle\mathbf{p}, d \mathbf{q}\rangle+\left\langle\mathbf{p}, \rho\left(\mathrm{i}^{*} A\right) \mathbf{q}\right\rangle
\end{aligned}
$$

where $\mathbf{q}=\sum_{k=0}^{n^{\prime}+1} \mathbf{q}_{(k)}$ and $\mathbf{p}=\sum_{k=0}^{n^{\prime}+1} \mathbf{p}_{(k)}$ are the auxiliary superfields corresponding to $q$ and $p$; component $\mathbf{q}_{(k)}$ is a $R$-valued $k$-form on $\gamma$ with internal degree $m-k$ and $\mathbf{p}_{(k)}$ is a $R^{*}$-valued $k$-form on $\gamma$ of internal degree $n^{\prime}-m-k$.

Pre-observable (105) can be pushed forward to zero modes (in the sense of Proposition 2) as in Section 6.3:

$$
\begin{aligned}
& \mathcal{F}_{\gamma}^{\mathrm{Z}}=R[m] \otimes H^{\bullet}(\gamma) \oplus R^{*}\left[n^{\prime}-m\right] \otimes H^{\bullet}(\gamma), \quad \Omega_{\gamma}^{\mathrm{Z}}=\int_{\gamma}\left\langle\delta \mathbf{p}_{\mathrm{z}}, \delta \mathbf{q}_{\mathrm{z}}\right\rangle, \\
& S_{\gamma}^{\mathrm{Z}}=\int_{\gamma}\left\langle\mathbf{p}_{\mathrm{z}}, \rho\left(\mathrm{i}^{*} A\right)\left(\mathrm{id}+G \rho\left(\mathrm{i}^{*} A\right)\right)^{-1} \mathbf{q}_{\mathrm{z}}\right\rangle-i \log \operatorname{tor}\left(\gamma, \mathrm{i}^{*} A, \rho\right)
\end{aligned}
$$

where we introduced the notation

$$
\operatorname{tor}\left(\gamma, i^{*} A, \rho\right)=\exp \operatorname{tr}_{R} \sum_{k \geq 2} \frac{(-1)^{k}}{k} \int_{\operatorname{Conf}_{k}(\gamma)} \overleftarrow{\prod_{1 \leq j \leq k}} \pi_{j+1, j}^{*} \eta \cdot \pi_{j}^{*} \rho\left(i^{*} A\right)
$$

Notations $G, \eta, \pi$ are the same as in Section 6.3. We use harmonic representatives for zero modes $\mathbf{q}_{\mathrm{z}}, \mathbf{p}_{\mathrm{z}}$ in $\mathcal{F}_{\gamma}$.

Taking the BV integral over zero modes in (106), we obtain the observable for Chern-Simons theory on $\Sigma$ :

$$
O_{\gamma}=\int_{\mathcal{L}_{\mathrm{z}} \subset \mathcal{F}_{\gamma}^{z}} \sqrt{\mu_{\mathrm{z}}}\left|\mathcal{L}_{\mathrm{z}} \mathrm{e}^{i \int_{\gamma}\left\langle\mathbf{p}_{\mathrm{z}}, \rho\left(\mathrm{i}^{*} A\right)\left(\mathrm{id}+G \rho\left(\mathrm{i}^{*} A\right)\right)^{-1}\right.} \mathbf{q}_{\mathrm{z}}\right\rangle \cdot \operatorname{tor}\left(\gamma, \mathrm{i}^{*} A, \rho\right)
$$

with $\mathcal{L}_{\mathrm{Z}} \subset \mathcal{F}_{\gamma}^{\mathrm{Z}}$ a Lagrangian subspace and $\mu_{\mathrm{z}}$ a translation-invariant volume element on $\mathcal{F}_{\gamma}^{z}$. Proposition 6 implies that $O_{\gamma}$ is $Q_{\Sigma}$-closed and that deformations of gauge fixing induce $Q_{\Sigma}$-exact shifts in $O_{\gamma}$.

In case $n^{\prime}=0, m=1$, with $\gamma=S^{1}$, (108) can be evaluated explicitly ${ }^{27}$ :

$$
\begin{aligned}
O_{\gamma} & =\int_{R[1] \oplus R^{*}[-1]} d \mathbf{q}_{(0)}^{\mathrm{z}} d \mathbf{p}_{(0)}^{\mathrm{z}} \mathrm{e}^{i \oint_{\gamma}\left\langle\mathbf{p}_{(0)}^{\mathrm{z}}, \rho(\bar{A}) \mathbf{q}_{(0)}^{\mathrm{z}}\right\rangle} \cdot \operatorname{det}_{R}\left(\frac{\sinh \rho(\bar{A} / 2)}{\rho(\bar{A} / 2)}\right) \\
& =\operatorname{det}_{R}\left(\rho(W)^{1 / 2}-\rho(W)^{-1 / 2}\right)=\operatorname{det}_{R}\left(\rho(W)-\mathrm{id}_{R}\right)
\end{aligned}
$$

where $W=\mathcal{P} \exp \oint_{\gamma} i^{*} A_{(1)}$ is the holonomy of the ambient connection around $\gamma$ (since this requires the choice of a starting point on $\gamma$, one can think of $W$ as a group element defined modulo conjugation). We also denote $\bar{A}=\log W$.

${ }^{27}$ One trick to simplify the calculation is to use gauge-invariance of $O_{\gamma}$ to pick the constant representative of the gauge equivalence class of connection $i^{*} A$. 
In (109), we used the gauge-fixing Lagrangian (72). In particular, the gauge fixing for the integral over zero modes is

$$
\underbrace{\left(R[1] \oplus R^{*}[-1]\right) \otimes H^{0}(\gamma)}_{\mathcal{L}_{z}} \subset \underbrace{\left(R[1] \oplus R^{*}[-1]\right) \otimes H^{\bullet}(\gamma)}_{\mathcal{F}_{\gamma}^{Z}}
$$

Remark 14. Observable (109) is only interesting (not identically zero) for representations $\rho$ such that $\operatorname{det} \rho \not \equiv 0$, i.e., $\rho(x)$ does not have zero eigenvalue for $x$ in an open dense subset of $\mathfrak{g}$. In particular, (109) is identically zero for $\rho=$ ad the adjoint representation.

Remark 15. Observable (109) has the property that it only depends on $i^{*} A_{(1)}$ and not on the other components of the ambient field. Also, it is the only representative of its class in $Q_{\Sigma}$-cohomology which depends only on $\mathrm{i}^{*} A$, for degree reasons (since $C^{\infty}\left(\left.\mathcal{F}_{\Sigma}\right|_{\gamma}\right)$ is non-negatively graded; we denote $\left.\mathcal{F}_{\Sigma}\right|_{\gamma}=\operatorname{Map}(T[1] \gamma, \mathcal{M})$ the space of pull-backs of ambient fields to $\gamma$ ).

In case $n^{\prime}>0, O_{\gamma}$ defined by (108) generally depends on $i^{*} A_{(k)}$ for $0 \leq k \leq n^{\prime}+1$. Also, there is no canonical representative of the class of $O_{\gamma}$ in $Q_{\Sigma}$-cohomology and no canonical choice for the gauge-fixing Lagrangian $\mathcal{L} \subset \mathcal{F}_{\gamma}$.

For $n^{\prime}=-1$, convergence requirement for the integral over zero modes (108) forces (cf. Remark 12) $\mathcal{L}_{\mathrm{Z}}=\left(R[m] \oplus R^{*}[-1-m]\right)^{\text {odd }}$ which yields $O_{\gamma}=0$.

For $n^{\prime}=2$, for $\gamma$ a connected closed oriented 3-manifold with non-zero first Betti number, we may take $m=1$ and choose the gauge fixing for zero modes as

$$
\mathcal{L}_{\mathrm{Z}}=\left(R[1] \oplus R^{*}[1]\right) \otimes(H^{0}(\gamma) \oplus \underbrace{\lambda^{\perp}}_{\subset H^{1}(\gamma)} \oplus \underbrace{\lambda}_{\subset H^{2}(\gamma)})
$$

where $\lambda$ is any line in $H^{1}(\gamma)$ and $\lambda^{\perp}$ is its orthogonal complement in $H^{2}(\gamma)$ w.r.t. the Poincaré duality.

If the condition $\operatorname{det} \rho \not \equiv 0$ as in Remark 14 holds, $O_{\gamma}$ defined with zero mode gauge fixing (110) is well defined on an open subset of $\left.\mathcal{F}_{\Sigma}\right|_{\gamma}$ and is not identically zero.

In case $\operatorname{dim} H^{1}(\gamma)=1$, Lagrangian (110) is the same as in Remark 12, i.e., $\mathcal{L}_{Z}=$ $\left(\mathcal{F}_{\gamma}^{Z}\right)^{\text {odd }}$. In case $\operatorname{dim} H^{1}(\gamma)>1$, the Lagrangian $\left(\mathcal{F}_{\gamma}^{Z}\right)^{\text {odd }}$ yields $O_{\gamma}$ which is identically zero.

In case of $\gamma$, a rational homology sphere, $H^{1}(\gamma)=0$, construction (110) is not applicable. However, one can take

$$
\mathcal{L}_{\mathrm{Z}}=\left(R[1] \oplus R^{*}[1]\right) \otimes H^{0}(\gamma)
$$

which produces an observable $O_{\gamma}$ of degree $-2 \operatorname{dim} R$. 
Remark 16. In case $n^{\prime}=2$ and $\gamma=\Sigma, \mathrm{i}=\mathrm{id}$, property (63) does not generally hold, so (105) is a pre-observable in the sense of Definition 6 , but $S_{\Sigma}+S_{\gamma}$ does not satisfy the classical master equation (62) on the space $\mathcal{F}_{\Sigma} \times \mathcal{F}_{\gamma}$. On the level of observable $O_{\gamma}$ this means that $Q_{\Sigma} O_{\gamma}=0$, but $S_{\Sigma}-i \log O_{\gamma}$ does not satisfy the classical master equation on $\mathcal{F}_{\Sigma}$.

In case $n^{\prime}=1$, for $\gamma$ a connected closed oriented surface of genus $g>0$, we may take $m=0$ and set

$$
\mathcal{L}_{\mathrm{Z}}=R \otimes\left(H^{0}(\gamma) \oplus \lambda^{\perp}\right) \oplus R^{*}[1] \otimes\left(H^{0}(\gamma) \oplus \lambda\right)
$$

where $\lambda$ is again any line $H^{1}(\gamma)$ and $\lambda^{\perp}$ is its orthogonal in $H^{1}(\gamma)$ w.r.t. the Poincaré duality. Assuming $\operatorname{det} \rho \not \equiv 0$, with this choice of $\mathcal{L}_{\mathrm{Z}}$ we produce an observable $O_{\gamma}$ of degree $(2 g-2) \operatorname{dim} R$ which is well defined and non-zero on an open subset of $\left.\mathcal{F}_{\Sigma}\right|_{\gamma}$.

Expression (108) also is an observable for $B F$ theory in dimension $D$. In this case, $n^{\prime}$ should satisfy $-1 \leq n^{\prime} \leq D-1$. For $n^{\prime}=2 k$ and $\gamma$ a closed oriented submanifold of $\Sigma$ of dimension $2 k+1$, we may take $m=2 k+1$ and choose the gauge fixing $\mathcal{L}_{\mathrm{Z}}$ as

$$
\mathcal{L}_{\mathrm{Z}}=\left(R[2 k+1] \oplus R^{*}[-1]\right) \otimes \mathcal{L}^{\prime}
$$

where $\mathcal{L}^{\prime} \subset H^{\bullet}(\gamma)$ a Lagrangian subspace with the property

$$
\operatorname{dim} \mathcal{L}_{j}^{\prime}=\operatorname{dim} \mathcal{L}_{2 k-j}^{\prime}
$$

where $\mathcal{L}_{j}^{\prime}=\mathcal{L}^{\prime} \cap H^{j}(\gamma)$, which is equivalent to

$$
\operatorname{dim} \mathcal{L}_{j}^{\prime}=B_{j}-B_{j-1}+\cdots+(-1)^{j} B_{0}
$$

where $B_{j}=\operatorname{dim} H^{j}(\gamma)$. For $\mathcal{L}^{\prime}$ to exist, we require that for $\gamma$ the combinations of Betti numbers on the r.h.s. of (112) are non-negative. Assuming $\operatorname{det} \rho \neq 0$, property (111) ensures that the integral over zero modes in (108) exists and is non-zero in an open subset of $\left.\mathcal{F}_{\Sigma}\right|_{\gamma}$.

\subsection{A CODIMENSION 2 (PRE-)OBSERVABLE IN BF THEORY}

The following example is due to Cattaneo-Rossi [10]. Let $\mathcal{M}=\mathfrak{g}[1] \oplus \mathfrak{g}^{*}[D-2]$ with the structure of degree $D-1$ Hamiltonian $Q$-manifold as in (23). One constructs a trivial degree $D-3$ Hamiltonian $Q$-bundle over $\mathcal{M}$ with fiber data

$$
\begin{aligned}
& \mathcal{N}=\mathfrak{g} \oplus \mathfrak{g}^{*}[D-3], \quad \mathcal{A}=\left\langle[\psi, q], \frac{\partial}{\partial q}\right\rangle+\left\langle\operatorname{ad}_{\psi}^{*} p, \frac{\partial}{\partial p}\right\rangle+(-1)^{D}\left\langle\xi, \frac{\partial}{\partial p}\right\rangle, \\
& \omega^{\prime}=\langle\delta p, \delta q\rangle, \quad \alpha^{\prime}=\langle p, \delta q\rangle, \quad \Theta^{\prime}=\langle p,[\psi, q]\rangle+\langle\xi, q\rangle
\end{aligned}
$$


Here, $q$ is the $\mathfrak{g}$-valued coordinate of degree 0 on $\mathfrak{g}$ and $p$ is the $\mathfrak{g}^{*}$-valued coordinate of degree $D-3$ on $\mathfrak{g}^{*}[D-3] ; \psi$ and $\xi$ are coordinates on $\mathcal{M}$ as in Section 2.3.

Given a closed oriented $D$-manifold $\Sigma$ and a closed oriented submanifold i: $\gamma \hookrightarrow$ $\Sigma$ of codimension 2, by the construction of Section 5 we get a pre-observable for the $B F$ theory on $\Sigma$ associated to the Hamiltonian $Q$-bundle $(23,113)$ :

$$
\begin{aligned}
& \mathcal{F}_{\gamma}=\mathfrak{g} \otimes \Omega^{\bullet}(\gamma) \oplus \mathfrak{g}^{*}[D-3] \otimes \Omega^{\bullet}(\gamma), \\
& \Omega_{\gamma}=(-1)^{D} \int_{\gamma}\langle\delta \mathbf{p}, \delta \mathbf{q}\rangle, \\
& S_{\gamma}=\int_{\gamma}\langle\mathbf{p}, d \mathbf{q}\rangle+\left\langle\mathbf{p},\left[i^{*} A, \mathbf{q}\right]\right\rangle+\left\langle i^{*} B, \mathbf{q}\right\rangle
\end{aligned}
$$

where $\mathbf{q}=\sum_{k=0}^{D-2} \mathbf{q}_{(k)}$ and $\mathbf{p}=\sum_{k=0}^{D-2} \mathbf{p}_{(k)}$ are the auxiliary superfields corresponding to $q$ and $p ; \mathbf{q}_{(k)}$ and $\mathbf{p}_{(k)}$ are $k$-forms on $\gamma$ with values in $\mathfrak{g}$ and $\mathfrak{g}^{*}$, respectively, having internal degrees $-k$ and $D-3-k$, respectively. ${ }^{28}$

Push-forward of the pre-observable (114-116) to zero modes (in the sense of Proposition 2) is evaluated as in Section 6.3:

$$
\begin{aligned}
\mathcal{F}_{\gamma}^{\mathrm{Z}} & =\mathfrak{g} \otimes H^{\bullet}(\gamma) \oplus \mathfrak{g}^{*}[D-3] \otimes H^{\bullet}(\gamma), \quad \Omega_{\gamma}^{\mathrm{z}}=\int_{\gamma}\left\langle\delta \mathbf{p}_{\mathrm{z}}, \delta \mathbf{q}_{\mathrm{z}}\right\rangle, \\
S_{\gamma}^{\mathrm{Z}} & =\int_{\gamma}\left\langle\mathrm{i}^{*} B+(-1)^{D} \mathrm{ad}_{\mathrm{i}^{*} A}^{*} \mathbf{p}_{\mathrm{z}},\left(\mathrm{id}+G \mathrm{ad}_{\mathrm{i}^{*} A}\right)^{-1} \mathbf{q}_{\mathrm{z}}\right\rangle-i \log \operatorname{tor}\left(\gamma, \mathrm{i}^{*} A, \mathrm{ad}\right)
\end{aligned}
$$

where tor is as in (107), for $\rho=$ ad the adjoint representation of $\mathfrak{g}$.

In the case of abelian $B F$ theory, $\mathfrak{g}=\mathbb{R}$, (117) yields

$$
\mathrm{e}^{i S_{\gamma}^{\mathrm{z}}}=\mathrm{e}^{i \int_{\gamma}\left\langle i^{*} B, \mathbf{q}_{\mathbf{z}}\right\rangle}
$$

which is an observable if we understand $\mathbf{q}_{\mathrm{z}} \in H^{\bullet}(\gamma)$ as an external parameter (the crucial point here is that by construction (118) satisfies the semi-quantum master equation (43), but the last term in (43) vanishes since $S_{\gamma}^{\mathrm{Z}}$ depends only on $\mathbf{q}_{\mathrm{z}}$ ). Taking $\mathbf{q}_{\mathrm{z}}=c \cdot 1 \in H^{0}(\gamma)$, we obtain the observable (91).

In [10] it is shown that in the case of "long knots" $\mathbb{R}^{D-2} \sim \gamma \subset \Sigma=\mathbb{R}^{D}$, embeddings of $\mathbb{R}^{D-2}$ into $\mathbb{R}^{D}$ with prescribed linear asymptotics, the push-forward of pre-observable (114-116) to zero modes yields, upon fixing the values of zero modes to $\mathbf{q}_{\mathrm{z}}=c \otimes 1 \in \mathfrak{g}^{*} \otimes \Omega^{0}(\gamma)$ and $\mathbf{p}_{\mathrm{z}}=0$, an observable, generalizing (91) in nonabelian case.

${ }^{28}$ The original construction of [10] started directly from the auxiliary action (116) on the space (114) and the idea was to construct an observable by integrating out the auxiliary fields. The rigorous definition for the latter integral was given in the case of "long knots" $\mathbb{R}^{D-2} \hookrightarrow \mathbb{R}^{D}$ (see below). Our point is that we embed the construction of [10] into our more general framework, and we have a more systematic way to pass from pre-observables to observables via fiber BV integrals. The latter allows for more general spaces of zero modes than the case of long knots; we postpone the detailed discussion of the corresponding observable to a future paper. 


\section{Final Remarks}

In this paper, we presented a two-step construction of classical observables in AKSZ sigma models, which consists of: (i) constructing an extension of the AKSZ theory to a BV theory on a larger space of fields using an extension of the target to a Hamiltonian $Q$-bundle, (ii) using the $\mathrm{BV}$ push-forward to the original space of fields to produce an observable. We also provided some examples, and in particular recovered the well-known Wilson loop observable in Chern-Simons theory together with its path integral representation [2] and the Cattaneo-Rossi "Wilson surface" observable in $B F$ theory.

There are natural questions to this construction not answered here, to which we hope to return in a future publication:

- Extend the zoo of explicit examples. In particular,

- give more explicit examples of "Wilson loops" in Poisson sigma model, Section 7.3 (which requires a solution of (99) as the input for step (i) of the construction and its quantization satisfying (102) for step (ii)) other than those mentioned in Remark 13;

- give an example corresponding to a non-trivial Hamiltonian $Q$-bundle over the target of the AKSZ sigma model.

- Calculate the isotopy invariants of embeddings given by expectation values of our observables.

- Study the application to constructing higher cocycles of the spaces of embeddings.

\subsection{EXTENSION TO SOURCE MANIFOLDS WITH BOUNDARY}

Our construction of observables possesses an extension to the case when the source manifold $\Sigma$ has a boundary $\partial \Sigma$, and the submanifold $\gamma \subset \Sigma$ on which the observable is supported is also allowed to have boundary, $\partial \gamma \subset \partial \Sigma$. We will outline this extension here, treating gauge theories with boundary in "BV-BFV formalism" [9]. For a more detailed example, Chern-Simons theory on a manifold with boundary with Wilson lines ending on the boundary, the reader is referred to [3]. We plan to give a more detailed treatment and more examples of the general construction in a future publication.

Recall [9] that a gauge theory on manifold $\Sigma$ with boundary $\partial \Sigma$ in BV-BFV formalism is described by the following data:

- A degree 0 Hamiltonian $Q$-manifold $\left(\mathcal{F}_{\partial \Sigma}, Q_{\partial \Sigma}, \Omega_{\partial \Sigma}=\delta \alpha_{\partial \Sigma}, S_{\partial \Sigma}\right)$ - "the BFV phase space" (or "the space of boundary fields") associated to the boundary $\partial \Sigma$.

- A $Q$-manifold $\left(\mathcal{F}_{\Sigma}, Q_{\Sigma}\right)$ ("the space of bulk fields") endowed with a $Q$ morphism $\pi: \mathcal{F}_{\Sigma} \rightarrow \mathcal{F}_{\partial \Sigma}$ (restriction of fields to the boundary), a degree -1 symplectic form $\Omega_{\Sigma}$ and a degree 0 action $S_{\Sigma} \in C^{\infty}\left(\mathcal{F}_{\Sigma}\right)$ satisfying

$$
\delta S_{\Sigma}=\iota_{Q_{\Sigma}} \Omega_{\Sigma}+\pi^{*} \alpha_{\partial \Sigma}
$$


Equation (119) replaces the condition that $S_{\Sigma}$ is the Hamiltonian function for $Q_{\Sigma}$ in Definition 4, and its consequence $Q_{\Sigma} S_{\Sigma}=\pi^{*}\left(\iota_{Q_{\partial \Sigma}} \alpha_{\partial \Sigma}-2 S_{\partial \Sigma}\right)$ or, equivalently,

$$
\frac{1}{2} \underbrace{\left\{S_{\Sigma}, S_{\Sigma}\right\}_{\Omega_{\Sigma}}}_{:=l_{Q_{\Sigma}} \varrho_{Q_{\Sigma}} \Omega_{\Sigma}}+\pi^{*} S_{\partial \Sigma}=0
$$

replaces the classical master equation.

AKSZ sigma models on manifolds with boundary fit naturally into BV-BFV formalism: one takes the standard construction $(8,9,15,16)$ for the bulk and sets

$$
\begin{aligned}
\mathcal{F}_{\partial \Sigma}=\operatorname{Map}(T[1] \partial \Sigma, \mathcal{M}), & Q_{\partial \Sigma}=d_{\partial \Sigma}^{\text {lifted }}+Q^{\text {lifted }}, \quad \Omega_{\partial \Sigma}=\tau_{\partial \Sigma}(\omega), \\
\alpha_{\partial \Sigma}=(-1)^{\operatorname{dim} \Sigma-1} \tau_{\partial \Sigma}(\alpha), & S_{\partial \Sigma}=(-1)^{\operatorname{dim} \Sigma-1}\left(\iota_{\partial \Sigma}^{\text {lifted }} \tau_{\partial \Sigma}(\alpha)+\tau_{\partial \Sigma}(\Theta)\right)
\end{aligned}
$$

for the boundary; $Q$-morphism $\pi: \operatorname{Map}(T[1] \Sigma, \mathcal{M}) \rightarrow \operatorname{Map}(T[1] \partial \Sigma, \mathcal{M})$ is the pull-back by the inclusion $\partial \Sigma \hookrightarrow \Sigma$.

A classical observable for a gauge theory in BV-BFV formalism (generalizing Definition 5) can be defined as the following collection of data.

- A graded vector space (the auxiliary space of states) $\mathcal{H}_{\partial \gamma}$ and a degree 1 element

$$
\delta_{\partial \gamma} \in C^{\infty}\left(\mathcal{F}_{\partial \Sigma}\right) \otimes \operatorname{End}\left(\mathcal{H}_{\partial \gamma}\right)
$$

satisfying

$$
Q_{\partial \Sigma} \delta_{\partial \gamma}+\delta_{\partial \gamma}^{2}=0
$$

- A degree $0 \mathcal{H}_{\partial \gamma}$-valued function of bulk fields

$$
O_{\gamma} \in C^{\infty}\left(\mathcal{F}_{\Sigma}\right) \otimes \mathcal{H}_{\partial \gamma}
$$

satisfying

$$
\left(Q_{\Sigma}+\pi^{*} \delta_{\partial \gamma}\right) O_{\gamma}=0
$$

The locality requirement is that $O_{\gamma}$ only depends on the restrictions of bulk fields to $\gamma$; likewise, $\delta_{\partial \gamma}$ should only depend on the restrictions of boundary fields to $\partial \gamma$.

We can also define a pre-observable in the BV-BFV context as the following data.

- A degree 0 Hamiltonian $Q$-bundle over $\left(\mathcal{F}_{\partial \Sigma}, Q_{\partial \Sigma}\right)$ with fiber data $\left(\mathcal{F}_{\partial \gamma}\right.$, $\left.\mathcal{A}_{\partial \gamma}, \Omega_{\partial \gamma}=\delta \alpha_{\partial \gamma}, S_{\partial \gamma}\right)$.

- A trivial $Q$-bundle over $\left(\mathcal{F}_{\Sigma}, Q_{\Sigma}\right)$ with fiber data $\left(\mathcal{F}_{\gamma}, \mathcal{A}_{\gamma}\right)$, with projection $\bar{\pi}$ : $\mathcal{F}_{\gamma} \rightarrow \mathcal{F}_{\partial \gamma}$ such that $d \bar{\pi}\left(\mathcal{A}_{\gamma}\right)=\mathcal{A}_{\partial \gamma}$, with fiber degree -1 symplectic structure $\Omega_{\gamma}$ and with degree 0 auxiliary action $S_{\gamma} \in C^{\infty}\left(\mathcal{F}_{\Sigma} \times \mathcal{F}_{\gamma}\right)$ satisfying

$$
\delta^{\mathrm{vert}} S_{\gamma}=\iota_{\mathcal{A}_{\gamma}} \Omega_{\gamma}+\bar{\pi}^{*} \alpha_{\partial \gamma}, \quad Q_{\Sigma} S_{\gamma}+\frac{1}{2} \underbrace{\left\{S_{\gamma}, S_{\gamma}\right\}_{\Omega_{\gamma}}}_{:=\iota_{\mathcal{A}} \iota_{\mathcal{A}} \iota_{\mathcal{\gamma}} \Omega_{\gamma}}+\pi_{\text {tot }}^{*} S_{\partial \gamma}=0
$$


where $\delta^{\text {vert }}$ is the de Rham differential on $\mathcal{F}_{\gamma}$ and $\pi_{\text {tot }}=\pi \times \bar{\pi}: \mathcal{F}_{\Sigma} \times \mathcal{F}_{\gamma} \rightarrow \mathcal{F}_{\partial \Sigma} \times$ $\mathcal{F}_{\partial \gamma}$.

Passing from a pre-observable to observable is a quantization problem. We construct $\mathcal{H}_{\partial \gamma}$ as the geometric quantization of the symplectic manifold $\left(\mathcal{F}_{\partial \gamma}, \Omega_{\partial \gamma}\right)$ and $\delta_{\partial \gamma}$ as the geometric quantization of $S_{\partial \gamma}$ :

$$
\mathcal{H}_{\partial \gamma}=\operatorname{GeomQuant}\left(\mathcal{F}_{\partial \gamma}, \Omega_{\partial \gamma}\right), \quad \delta_{\partial \gamma}=i \hat{S}_{\partial \gamma}
$$

For simplicity, assume that the Lagrangian polarization of $\mathcal{F}_{\partial \Sigma}$ used for the geometric quantization is the vertical polarization of a fibration $\mathrm{p}: \mathcal{F}_{\partial \Sigma} \rightarrow \mathcal{B}$. Then $O_{\gamma}$ is given by the following modification of the fiber $\mathrm{BV}$ integral:

$$
O_{\gamma}(X, b)=\int_{\mathcal{L}_{b} \subset \bar{\pi}^{-1} \mathrm{p}^{-1} b} \mathcal{D} Y \mathrm{e}^{i S_{\gamma}(X, Y)}
$$

for $b \in \mathcal{B}, X \in \mathcal{F}_{\Sigma} ; Y$ runs over $\mathcal{F}_{\gamma} ; \mathcal{L}_{b}$ is a Lagrangian in $\bar{\pi}^{-1} \mathrm{p}^{-1} b \subset \mathcal{F}_{\gamma}$.

Given an AKSZ theory on $\Sigma$ with target $\mathcal{M}$ and a Hamiltonian $Q$-bundle over $\mathcal{M}$, as a first step, we construct a pre-observable with the bulk data given by old formulae (54) and boundary data given by the same formulae with $\gamma$ replaced by $\partial \gamma:$

$$
\begin{aligned}
& \mathcal{F}_{\partial \gamma}=\operatorname{Map}(T[1] \partial \gamma, \mathcal{N}), \quad \mathcal{A}_{\partial \gamma}=d_{\partial \gamma}^{\text {lifted }}+p_{\partial}^{*} \mathcal{A}^{\text {lifted }}, \quad \Omega_{\partial \gamma}=\tau_{\partial \gamma}\left(\omega^{\prime}\right), \\
& \alpha_{\partial \gamma}=(-1)^{\operatorname{dim} \gamma-1} \tau_{\partial \gamma}\left(\alpha^{\prime}\right), \quad S_{\partial \gamma}=(-1)^{\operatorname{dim} \gamma-1}\left(\iota_{d_{\partial \gamma}^{\text {lifted }}} \alpha_{\partial \gamma}+p_{\partial}^{*} \tau_{\partial \gamma}^{\text {tot }}\left(\Theta^{\prime}\right)\right)
\end{aligned}
$$

where $p_{\partial}: \operatorname{Map}(T[1] \partial \Sigma, \mathcal{M}) \rightarrow \operatorname{Map}(T[1] \partial \gamma, \mathcal{M})$ is the restriction of ambient boundary fields to $\partial \gamma$. As the second step, we pass from this pre-observable to an observable using construction $(120,121)$.

\section{Acknowledgements}

I wish to thank Anton Alekseev, Alberto Cattaneo, Andrei Losev and Nicolai Reshetikhin for inspiring discussions. This work was partially supported by RFBR Grant 11-01-00570-a and by SNF Grant 200021_137595.

\section{References}

1. Aleksandrov, M., Kontsevich, M., Schwarz, A., Zaboronsky, O.: The geometry of the master equation and topological quantum field theory. Int. J. Mod. Phys. A 12, 14051430 (1997)

2. Alekseev, A., Faddeev, L., Shatashvili, S.: Quantization of symplectic orbits of compact Lie groups by means of the functional integral. J. Geom. Phys. 5(3), 391-406 (1988)

3. Alekseev, A., Barmaz, Y., Mnev, P.: Chern-Simons theory with Wilson lines and boundary in the BV-BFV formalism. J. Geom. Phys. 67, 1-15 (2013). arXiv:1212.6256 (math-ph) 
4. Axelrod, S., Singer, I.M.: Chern-Simons perturbation theory. I. Perspectives in mathematical physics, pp. 17-49, Conf. Proc. Lecture Notes Math. Phys., vol. III. Int. Press, Cambridge (1994)

5. Axelrod, S., Singer, I.M.: Chern-Simons perturbation theory. II. J. Differ. Geom. 39, 173-213 (1994)

6. Bonechi, F., Qiu, J., Zabzine, M.: Wilson Lines from Representations of NQ-Manifolds. arXiv:1108.5358 (math.DG)

7. Bott, R., Taubes, C.: On the self-linking of knots. J. Math. Phys. 35, 5247-5287 (1994)

8. Cattaneo, A.S., Felder, G.: On the AKSZ formulation of the Poisson sigma model. Lett. Math. Phys. 56, 163-179 (2001)

9. Cattaneo, A.S., Mnev, P., Reshetikhin, N.: Classical BV theories on manifolds with boundaries. arXiv:1201.0290 (math-ph)

10. Cattaneo, A.S., Rossi, C.: Wilson surfaces and higher dimensional knot invariants. Commun. Math. Phys. 256, 513-537 (2005)

11. Costello, K.: Renormalization and effective field theory, Mathematical Surveys and Monographs, vol. 170. AMS, Providence (2011)

12. Kotov, A., Strobl, T.: Characteristic classes associated to Q-bundles. arXiv:0711.4106 (math.DG)

13. Losev, A.: Lectures on topological quantum field theories (2008)

14. Mnev, P.: Discrete $B F$ theory. arXiv:0809.1160 (hep-th)

15. Qiu, J., Zabzine, M.: Knot invariants and new weight systems from general 3D TFTs. J. Geom. Phys. 62(2), 242-271 (2012)

16. Roytenberg, D.: AKSZ-BV formalism and Courant algebroid-induced topological field theories. Lett. Math. Phys. 79, 143-159 (2007)

17. Schwarz, A.S.: The partition function of a degenerate functional. Commun. Math. Phys. 67(1), 1-16 (1979)

18. Schwarz, A.S.: Geometry of Batalin-Vilkovisky quantization. Commun. Math. Phys. 155, 249-260 (1993)

19. Yu Vaintrob, A.: Lie algebroids and homological vector fields. 1997 Russ. Math. Surv. 52, 428

20. Witten, E.: Quantum field theory and the Jones polynomial. Commun. Math. Phys. 121, 351-399 (1989) 